Zur Rolle der Ökonometrie in der wissenschaftlichen Politikberatung

Gebhard Kirchgässner

November 2012 Discussion Paper no. 2012-23 
Editor:

Publisher:

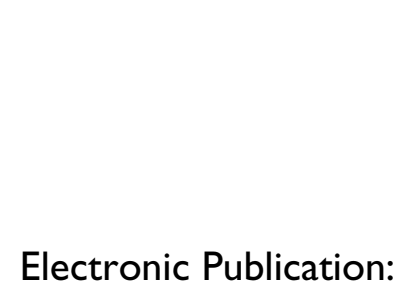

Martina Flockerzi

University of St. Gallen

School of Economics and Political Science

Department of Economics

Bodanstrasse 8

$\mathrm{CH}-9000$ St. Gallen

Phone +41712242325

Fax +41712243135

Email_seps@unisg.ch

School of Economics and Political Science

Department of Economics

University of St. Gallen

Bodanstrasse 8

$\mathrm{CH}-9000$ St. Gallen

Phone $\quad+41712242325$

Fax +41712243135

http://www.seps.unisg.ch 


\title{
Zur Rolle der Ökonometrie \\ in der wissenschaftlichen Politikberatung ${ }^{1}$
}

\author{
Gebhard Kirchgässner²
}

Author's address: $\quad$ Prof. Dr. Gebhard Kirchgässner

SIAW-HSG

Bodanstrasse 8

$\mathrm{CH}-9000$ St. Gallen

Phone +41712242340

Fax +41712242298

Email gebhard.kirchgaessner@unisg.ch

Website www.siaw.unisg.ch

\footnotetext{
1 Thünen-Vorlesung 2012 des Vereins für Socialpolitik, Göttingen, 10. September 2012. Überarbeitete schriftliche Fassung, November 2012. Erscheint in: Perspektiven der Wirtschaftspolitik. - @ Gebhard Kirchgässner.

2 Universität St. Gallen, Schweizerisches Institut für Aussenwirtschaft und Angewandte Wirtschaftsforschung, Leopoldina und CESifo
} 


\begin{abstract}
Because economic theory alone does in many situations not provide unambiguous policy advice, most of the time empirical analyses are needed in addition. Thus, today econometric analyses are often parts of reports for political institutions or courts. However, it is not unusual that reports with contradicting evidence are presented by different groups or parties. Using the relation between government size and economic growth as an example, it is shown how such contradicting results are possible even if all scientists involved behave sincerely and adhere to the rules of scientific research. Our second example, studies investigating whether the death penalty serves as a deterrent to homicide, shows that the results of empirical analyses might to a large extent depend on a priori convictions of the scientists. Thus, the process of scientific policy advice has to be organised in a way so that similar to the genuinely scientific discourse - open discussion and criticisms of methods and results are possible. In order to disclose possible conflicts of interests, this demand transparency of the whole process and, in particular for empirical analyses, that data and programmes are made available for re-estimations.
\end{abstract}

\title{
Keywords
}

Economic Policy Advice, Economic Theory, Econometrics, Size of Government, Death Penalty, Objectivity, Self-Interest.

\section{JEL Classification}

C10, H11, H19, K14. 
Auf der Tagung des Vereins für Socialpolitik im Jahr 1978 in Hamburg ergab sich im Anschluss an ein Plenumsreferat von Olaf Sievert über „Die Steuerbarkeit der Konjunktur durch den Staat" ein Disput zwischen ihm und Hans-Jürgen Krupp über die Frage, ob die damalige Arbeitslosigkeit in der Bundesrepublik Deutschland keynesianischer oder nicht-keynesianischer Natur sei. Der damalige Vorsitzende, Wilhelm Krelle, griff in diese Diskussion ein und bemerkte, die Kollegen müssten darüber nicht streiten. Schließlich gebe es die Ökonometrie, mit deren Hilfe man diese Frage entscheiden könne. ${ }^{1}$

Trotz erheblichen Einsatzes der Ökonometrie und eines starken Ausbaus dieser Teildisziplin an den Universitäten in den vergangenen Jahrzehnten werden solche Diskussionen bis heute immer wieder geführt. Offensichtlich lassen sich solche Fragen nicht ganz so einfach beantworten, wie dies Wilhelm Krelle seinerzeit vorschwebte. Andererseits kann man feststellen, dass ökonometrische Analysen heute in vielen Bereichen auch und gerade in der Politikberatung unverzichtbar geworden sind. Dies gilt z.B. für die Zentralbanken: Sie könnten ihre Geldpolitik ohne solche Analysen kaum mehr durchführen; man denke nur an die Prognosen der Preisentwicklung, die zur Festlegung der Referenzzinssätze erforderlich sind. ${ }^{2}$ Man könnte es sich daher einfach machen und darauf hinweisen, wie nützlich ökonometrische Arbeiten für viele Politikbereiche geworden sind, nicht nur für die Geld-, sondern z.B. auch für die Arbeitsmarkt- ${ }^{3}$ oder für die Wettbewerbspolitik. ${ }^{4}$ Nicht umsonst gibt es in Deutschland eine Reihe von - wesentlich aus öffentlichen Mitteln finanzierten - Wirtschaftsforschungsinstituten, die für ihre Analysen in aller Regel moderne ökonometrische Methoden verwenden.

Leider sind die Ergebnisse ökonometrischer Studien häufig nicht so eindeutig und abgesichert, wie man sich dies wünschen würde. Das gilt bereits für den ,handwerklichen` Bereich, wie aus einer Untersuchung des Journal of Money, Credit, and Banking zu Beginn der achtziger Jahre hervorgeht. ${ }^{5}$ Die Autoren von insgesamt 154 Arbeiten, die zwischen 1980 und 1982 erschienen oder eingereicht und entweder bereits akzeptiert oder noch im Begutachtungsprozess waren, wurden gebeten, ihre Daten und Programme zur Verfügung zu stellen. Nur von 54 Arbeiten wurden die Daten zur Verfügung gestellt, und nur in acht Fällen konnten die Ergebnisse ohne Probleme reproduziert werden. Eine Wiederholung dieses Projekts in den

1. Die Wiedergabe der Diskussion im Anschluss an das Referat von Sievert (1979) beruht auf eigenen Erinnerungen. In der schriftlichen Fassung des Schlussworts der Tagung von Krelle liest sich dies folgendermaßen: „Haben wir aber nun keynesianische oder nicht-keynesianische Arbeitslosigkeit? Das sollte nicht durch Bekenntnisse, sondern durch Tests geklärt werden. Wir können ja die wichtigsten Verhaltensfunktionen in der Volkswirtschaft schätzen und damit die Zusammenhänge zwischen Geldlöhnen, Preisen, Produktion und Beschäftigung und anderen Größen mit einiger Sicherheit feststellen.“ (1979, S. 853.)

2. Zur Anwendung ökonometrischer verfahren in der Geldpolitik siehe z.B. die Arbeiten in Heft 1/2010 der Schweizerischen Zeitschrift für Volkwirtschaft und Statistik, in dem Beiträge eines Symposiums aufgeführt sind, welches im Oktober 2009 in Zürich zum Thema „10 Jahre neue Geldpolitik der Schweizerischen Nationalbank" durchgeführt wurde.

3. Siehe hierzu z.B. Lechner (1989) oder Lechner und Gerfin (2000).

4. Siehe hierzu z.B. Duso, Neven und Röller (2007).

5. Siehe Dewald, Thursby und Anderson (1986). 
neunziger Jahren brachte ganz ähnliche Ergebnisse: Wiederum sahen sich jene, deren empirische Arbeiten etwas länger zurücklagen, nur teilweise in der Lage, ihre Daten und Programme zur Verfügung zu stellen. ${ }^{6}$

Dies zeigt, wie wichtig es ist, dass Daten und Programme veröffentlichter Arbeiten für Nachschätzungen zur Verfügung stehen. Dies wird auch deutlich an einem neueren Beispiel, der Kontroverse über die Auswirkungen des (illegalen) Herunterladens von Musik aus dem Internet auf den Absatz von CDs. Während davor (und auch danach) die meisten Studien zum Ergebnis kamen, dass das Herunterladen von Musik erhebliche negative Auswirkungen auf die Verkäufe von CDs hat, ${ }^{7)}$ kamen Oberholzer-Gee und Strumpf (2007) zum Ergebnis, dass das Herunterladen von Musik bestenfalls vernachlässigbar geringe negative Auswirkungen auf den Verkauf von CDs habe; der Rückgang dieser Verkäufe hat nach ihrer Ansicht andere Ursachen. Dieses Ergebnis, welches auch dem üblichen Common Sense widerspricht, ist insbesondere angesichts des parallel zum Auftauchen der Tauschbörsen starken Rückgangs der CD-Verkäufe nicht einfach nachvollziehbar. So ist es auch nicht erstaunlich, dass diese Arbeit - insbesondere von Liebowitz $(2007,2011)$ - scharf kritisiert wurde.

Die Frage ist nicht, wer in diesem Disput Recht hat; diese Frage ist auch kaum zu klären, da die Daten von Oberholzer-Gee und Strumpf (2007), die für eine kritische Analyse ihrer Ergebnisse erforderlich wären, nicht verfügbar sind: Die Autoren weigern sich, sie für eine solche Prüfung herauszugeben, weil sie vertraulich seien. Dazu kommt, dass das Journal of Political Economy, in welchem diese Arbeit publiziert wurde, nicht nur die Veröffentlichung eines Kommentars von Liebowitz abgelehnt hat, sondern dass, nachdem ein erster Referee darüber positiv geurteilt hatte, der Herausgeber dieser Zeitschrift, Steven Levitt, Strumpf, d.h. einen der beiden Autoren, als zweiten Referee beauftragt hat, was den Kriterien einer fairen Beurteilung ins Gesicht schlägt. Er rechtfertigt dies damit, dass es im Ermessen eines Herausgebers stehe, sowohl die Referees auszuwählen als auch letztlich über die Publikation zu entscheiden. ${ }^{8}$

Dieser Fall ist aber auch aus der Perspektive der Politikberatung interessant. In Stockholm fand in den Jahren 2009 und 2010 ein Prozess gegen die drei Betreiber der Internettauschbörse ,The Pirate Bay' sowie einen Geschäftsmann statt, der diese finanziell und logistisch unterstützt hatte, der ,Piratenbucht-Fall` (Pirate Bay Case). In diesem Prozess wurden sowohl die Arbeit von Oberholzer-Gee und Strumpf (2007) als auch die Arbeiten von Liebowitz (2006, 2007) von den jeweiligen Seiten als Evidenz für die Schädlichkeit bzw. Unschädlichkeit des illegalen Herunterladens herangezogen. Die vier Beschuldigten wurden freilich trotz der vorgebrachten Ergebnisse von Oberholzer-Gee und Strumpf (2007) sowohl in der ersten als auch

6. Siehe hierzu Anderson und Dewald (1994, S. 82f.).

7. Siehe hierzu die Übersicht in Liebowitz (2011, S. 14).

8. Siehe hierzu N. Häring, Steven Levitt Blocks an Undesired Statement: No Comment Please, Handelsblatt. online vom 23. Juni 2008

http://www.handelsblatt.com/politik/oekonomie/nachrichten/steven-levitt-blocks-an-undesired-statementno-comment-please/2976444.html (30/08/12) 
in der Rekursinstanz wegen Urheberrechtsverletzungen schuldig gesprochen und zu (unbedingten) Gefängnisstrafen sowie zu Schadensersatzzahlungen verurteilt. ${ }^{9}$

Dass Wissenschaftler vor Gerichten gelegentlich unterschiedliche Auffassungen vertreten, dürfte kaum überraschen. Dies gilt umso mehr, je mehr bei einer solchen Verhandlung auf dem Spiel steht. Wie dieser Fall zeigt, kann sich die Diskussion zwischen beiden Seiten auch um methodische Probleme der Ökonometrie handeln, und es können auch Arbeiten herangezogen werden, die in hochrangigen wissenschaftlichen Zeitschriften veröffentlicht wurden. In diesem Fall ist bzw. war für den Außenstehenden freilich nicht klar, inwieweit es dadurch mögliche Interessenkonflikte gegeben hat, dass die Autoren mit den jeweiligen Seiten in Verbindung standen. Dies ist anders, wenn Wissenschaftler direkt im Auftrag für politische Instanzen und/oder Interessengruppen Gutachten verfassen, deren Zweck es ist, bestimmte politische Ziele zu erreichen. Dies war z.B. bei der Diskussion um die kantonalen Monopolversicherungen gegen Feuer- und Elementarschäden in der Schweiz in den neunziger Jahren der Fall.

Es gibt in 19 Kantonen solche kantonalen Monopole, während in sieben Kantonen Feuer- und Elementarschäden durch private Versicherungen abgedeckt werden, die in Wettbewerb zueinander stehen. Wäre die Schweiz dem EWR beigetreten, hätten diese Monopole vermutlich aufgelöst werden müssen. Nach der Ablehnung des Beitritts zum Europäischen Wirtschaftsraum (EWR) im Dezember 1992 fand in der Schweiz eine Diskussion darüber statt, ob die Monopole der Kantonalen Gebäudeversicherungen beibehalten oder abgeschafft werden sollten; die schweizerischen Privatversicherer wollten trotz der Ablehnung des EWR deren Abschaffung. Um dem etwas entgegenzusetzen, beauftragten die Kantonalen Gebäudeversicherungen Thomas von Ungern-Sternberg (2005) von der Universität Lausanne mit der Erstellung eines Gutachtens, welches zeigen sollte, dass ihre Prämien deutlich tiefer als jene der privaten Konkurrenz sind. Nachdem er dies in seinem Gutachten dokumentiert hatte und es der Öffentlichkeit vorgestellt worden war, beauftragten die Privatversicherer Bernd Schips (2005) von der ETH Zürich mit der Erstellung eines Gegengutachtens. Er kam - wenig überraschend - zum gegenteiligen Ergebnis.

Das interessante an diesem Beispiel ist nicht, dass zwei Wissenschaftler zum gleichen Gegenstand unterschiedliche, sich widersprechende Auffassungen vertreten, sondern vielmehr, dass dies bei einer Frage geschieht, bei der theoretische Überlegungen nur eine vergleichsweise geringe Rolle spielen. Wird - wie seinerzeit im Hamburg - über die Ursachen der Arbeitslosigkeit diskutiert, kann man von unterschiedlichen theoretischen Vorstellungen ausgehen und damit auch zu unterschiedlichen Ergebnissen kommen. Bei der Frage der kantonalen Monopolversicherungen aber handelt es sich um eine sehr einfache Frage: Wer verlangt für die gleiche Leistung höhere Prämien: die kantonalen Monopole oder die Privatversicherungen. Hier stehen nicht große konkurrierende Theorien miteinander in Widerstreit, und die ökonometrischen Methoden, die für eine solche Untersuchung erforderlich sind, sind auch nicht

9. Verfahren B 13301-06. Zu diesem Fall siehe Göcke (2009), Manner, Siniketo und Polland (2009) sowie http://en.wikipedia.org/wiki/The_Pirate_Bay_trial (30/08/12) 
extrem aufwendig. Daher ist es umso merkwürdiger, dass die beiden Gutachter zu so unterschiedlichen Ergebnissen kamen, die - selbstverständlich rein zufällig - genau dem entsprachen, was die Auftraggeber von ihnen erwartet hatten. Dazu kommt, dass das Ergebnis von v. Ungern-Sternberg (2005), welches letztlich auch bestätigt wurde, ${ }^{10}$ im Widerspruch zur Auffassung mancher - oder vielleicht auch vieler - Ökonomen steht, die davon ausgehen, dass privater Wettbewerb immer und überall staatlichen Monopolen überlegen ist. ${ }^{11}$

Wenn man sich mit der Rolle der Ökonometrie in der wissenschaftlichen Politikberatung nicht nur bei wirtschaftspolitischen Fragestellungen - befassen will, macht es daher Sinn, sich auf jene Fälle zu konzentrieren, in denen verschiedene Wissenschaftler deutlich unterschiedliche Positionen vertreten, die sie mit Hilfe ökonometrischer Analysen zu untermauern suchen. Dass unterschiedliche Positionen auch von Wissenschaftlern mit hoher Reputation vertreten werden, ist Allgemeingut; man denke nur an die Minderheitsgutachten im deutschen Sachverständigenrat. Dass solche Unterschiede auch ideologisch bedingt sein könnten, hat Potrafke (2012) gezeigt: Minderheitsvoten kommen deutlich häufiger von jenen Mitgliedern des Sachverständigenrats, die von den Gewerkschaften oder den Arbeitsgebern benannt werden, wobei der Einfluss der Gewerkschaften deutlich grösser als jener der Arbeitgeber zu sein scheint. Der Einfluss aller anderen erklärenden Variablen, die er in seine Analyse einbezieht, ist weit von jeder statistischen Signifikanz entfernt. Wirklich interessant sind solche Unterschiede für unser Thema jedoch nur dann, wenn, wie z.B. im Fall des illegalen Herunterladens von Musik, die entsprechenden Aussagen mit Hilfe ökonometrischer Untersuchungen untermauert werden.

Dass der Ökonometrie bei der Beantwortung vieler Fragen eine zentrale Rolle zukommt, hängt mit der weitgehenden Unmöglichkeit zusammen, allein auf Theorie basierend eindeutige Aussagen zu machen. ${ }^{12}$ Wir sind in der Theorie oft hervorragend in der Lage, partielle Aussagen zu machen, d.h. Aussagen, die sich auf das Zusammenwirken weniger, aber wesentlicher wirtschaftlicher Variablen beziehen. Sehr viel schwieriger sind Aussagen, die ein allgemeines Gleichgewicht unterstellen. Hier sind in aller Regel sehr starke zusätzliche Annahmen erforderlich. Hält man sich an den Kern unserer Theorie und sieht man von solchen restriktiven Annahmen ab, dann kann man, wie Sonnenschein (1972, 1973), gezeigt hat, noch nicht einmal sicherstellen, dass ökonomische Systeme stabil und nicht chaotisch sind und dass Nachfragekurven global negativ geneigt sind und nicht lokal positive Steigungen existieren. Viel relevanter aber dürfte sein, dass in sehr vielen, politisch ausgesprochen relevanten Fällen, Einkommens- und Substitutionseffekt gegenläufig sind und man keine Aussage darüber treffen kann, welcher dieser beiden Effekte dominiert. Wird auf den Kaufkrafteffekt von Löhnen hingewiesen, unterstellt man üblicherweise, dass der Einkommenseffekt dominiert, während man dann, wenn man von einer Lohnsteigerung eine Beeinträchtigung der Wettbewerbsfähigkeit einer Branche oder eines Landes befürchtet, von der Dominanz des Substitutionsef-

10. Siehe hierzu auch Kirchgässner (1993).

11. Siehe hierzu Kirchgässner (1996).

12. Siehe z.B. die Ausführungen von Siebert (1998, S.62) unter Berufung auf John Stuart Mill (1848, S. 452) auf der Jahrestagung des Verein für Socialpolitik in Bern im Jahr 1997. 
fekts ausgeht. Analoges gilt für die zu erwartenden Auswirkungen einer ökologischen Steuerreform. Glaubt man, dass sie die Arbeitslosigkeit reduzieren würde, geht man wiederum von der Dominanz des Einkommenseffekts aus, während man dann den Substitutionseffekt als stärker ansieht, wenn man Arbeitsplatzverluste befürchtet.

Da die Politik entscheiden muss, muss sie in diesen Fällen implizit beurteilen, welchen Effekt sie als stärker ansieht. Hier sind empirische Untersuchungen kaum verzichtbar, weshalb die Statistik bzw. die Ökonometrie zwangsläufig ins Spiel kommt. Insofern hatte Wilhelm Krelle mit seiner oben angeführten Bemerkung durchaus einen wichtigen Punkt, auch wenn sein extrem starkes Vertrauen in die Gültigkeit mit Hilfe ökonometrischer Verfahren gewonnen Aussagen über die Wirklichkeit kaum haltbar sein dürfte.

Im Folgenden soll die Möglichkeit nahezu beliebiger Aussagen zunächst an einem Beispiel aus der Ökonomie aufgezeigt werden, dem Zusammenhang zwischen Staatsquote und Wirtschaftswachstum (Abschnitt 2). Als nächstes folgt ein Problem des Strafrechts: die umstrittene Abschreckungswirkung der Todesstrafe (Abschnitt 3). In diesem Bereich wenden heute nicht nur Ökonomen, sondern z.B. auch Juristen moderne ökonometrische Verfahren an. Der 4. Abschnitt geht auf den Prozess der Politikberatung ein. ${ }^{13}$ Die Arbeit schließt mit einer thesenartigen Zusammenfassung (Abschnitt 5).

\section{Der Zusammenhang zwischen Staatsquote und Wirtschaftswachstum}

$\mathrm{Ob}$ die Staatsquote zu hoch oder zu tief ist, ist ein alter politischer Streit. Es ist jedoch trivial und findet sich auch in Modellen endogenen Wachstums wie z.B. bei Barro (1990), dass es keinen linearen Zusammenhang zwischen der Staatsquote und der wirtschaftlichen Entwicklung geben kann. Eine Staatsquote von Null würde Anarchie bedeuten, was der wirtschaftlichen Entwicklung kaum zuträglich wäre. Das Gleiche gilt für eine Staatsquote, die hundert Prozent beträgt. Dazwischen muss irgendwo die ,optimale' Staatsquote liegen, d.h. in unserem Zusammenhang jene, die zu maximalem Wirtschaftswachstum führt. Wir haben somit eine Art ,Laffer-Kurve، für den Zusammenhang zwischen Staatsquote und wirtschaftlicher Entwicklung. Die einzige Frage, die man hier sinnvoll stellen kann, ist, ob die tatsächliche Staatsquote eines Landes höher oder niedriger als die optimale Quote ist. Im ersten Fall hätten wir lokal einen negativen, in letzterem einen positiven Zusammenhang zwischen Wirtschaftswachstum und Staatsquote. Diese Frage kann nicht theoretisch, sondern nur empirisch beantwortet werden.

Tatsächlich gibt es eine ganze Reihe von Studien, die zu unterschiedlichen Ergebnissen kamen, wobei freilich meistens ein negativer Zusammenhang festgestellt wurde. ${ }^{14}$ Wie beispielsweise in den Diskussionen zwischen Förster und Henrekson $(1999,2001,2006)$ auf der einen und Agell, Lindt und Ohlson $(1997,1999)$ bzw. Agell, Ohlson und Thoursie (2006) auf der anderen Seite deutlich wurde, ist das Problem vieler dieser Arbeiten, dass die Simultanei-

13. Sieh hierzu auch Kirchgässner (2011).

14. Eine Übersicht findet sich in Kirchgässner (2004). 
tät zwischen Staatsquote und Wirtschaftswachstum nicht oder zumindest nicht in ausreichendem Masse berücksichtigt wird. Geschätzt wird z.B. folgende Gleichung: ${ }^{15}$

$$
\mathrm{y}_{\mathrm{t}}-\mathrm{y}_{\mathrm{t}-1}=\beta_{0}+\beta_{1}\left(\mathrm{~g}_{\mathrm{t}}-\mathrm{y}_{\mathrm{t}}\right)+\beta_{2} \mathrm{y}_{\mathrm{t}-\mathrm{k}}+\sum_{\mathrm{j}=1}^{\mathrm{k}}\left(\beta_{\mathrm{j}+2} \mathrm{X}_{\mathrm{j}, \mathrm{t}}\right)+\varepsilon_{\mathrm{t}} .
$$

Dabei ist y der Logarithmus des realen Bruttoinlandsprodukts pro Kopf und g der Logarithmus der realen Staatsausgaben pro Kopf, d.h. links steht die reale Wachstumsrate pro Kopf und rechts die Staatsquote. Dazu kommen der um eine oder mehrere (k) Perioden verzögerte Logarithmus des realen Bruttoinlandsprodukts pro Kopf, $\mathrm{y}_{\mathrm{t}-\mathrm{k}}$, der die Konvergenz zum Wachstumspfad abbilden soll, weitere erklärende Variablen $\mathrm{X}_{\mathrm{j}}$ wie z.B. die Bevölkerung oder die Investititonsquote, von denen man annimmt, dass sie ebenfalls einen Einfluss auf das Wirtschaftswachstum haben, sowie ein stochastisches Restglied, $\varepsilon$, welches alle übrigen Einflüsse abdecken soll, die nicht explizit erfasst werden (können).

Da der verzögerte Wert des Bruttoinlandsprodukts im Zeitpunkt $t$ gegeben ist, wird dann, wenn die tatsächlichen Staatsausgaben den im Jahr zuvor festgelegten Budgetvorgaben entsprechen, jeder nicht erwartete Rückgang (Anstieg) zu einem Anstieg (Rückgang) der Staatsquote führen, und damit zu einem negativen Vorzeichen des geschätzten Parameters $\beta_{1}$. Dies gilt völlig unabhängig davon, wie der langfristige Zusammenhang zwischen Staatsquote und Wirtschaftswachstum aussieht. Dem kann man nur begegnen, indem man einen Instrumentenschätzer mit theoretisch gültigen und statistisch abgesicherten Instrumenten verwendet. Solche Instrumente sind freilich, wie die Literatur zeigt, schwer zu finden. Verzichtet man deshalb, wie z.B. Förster und Henrekson (2001), auf ein solches Schätzverfahren und verwendet die gewöhnliche Kleinst-Quadrate-Methode (OLS), negiert man das hier existierende Simultaneitätsproblem und produziert statistische Artefakte. Der von diesen beiden Autoren gemachte Verweis, dass diese negative Beziehung sich im Rahmen einer Extreme-BoundsAnalyse als sehr robust erweist, zeigt nur, dass diese kurzfristige negative Beziehung gegenüber alternativen Spezifikationen der Schätzgleichung sehr robust ist, besagt aber nichts über die eigentlich interessierende langfristige Beziehung.

Gelegentlich wird in diesen Schätzgleichungen nicht die Staats-, sondern die Steuerquote als erklärende Variable verwendet. ${ }^{16}$ Theoretisch sollte man annehmen, dass diese sehr viel eher einen negativen Einfluss auf die Wachstumsrate hat. Dass manche Studien zum Ergebnis kommen, dass die Steuern, wenn überhaupt, einen geringeren negativen Einfluss auf das Wirtschaftswachstum haben als die Staatsausgaben, dürfte damit zusammenhängen, dass die Steuereinnahmen schneller auf Konjunkturschwankungen reagieren als die Staatsausgaben.

Neben dem Problem, dass in diesen Studien lineare Beziehungen für einen Querschnitt über viele Länder mit sehr unterschiedlichen Staatsquoten geschätzt werden, was bei korrekter Spezifikation fast zwangsläufig zu nicht-signifikanten Ergebnissen führen muss, ergibt sich

15. Siehe z.B. Schaltegger (2004) oder Schaltegger and Torgler (2006).

16. Siehe z.B. Schaltegger (2004). 
ein weiteres Problem daraus, dass selbst dann, wenn man sich auf die OECD-Staaten beschränkt, für Staaten mit sehr unterschiedlichen Sozialsystemen und damit auch sehr unterschiedlichen Anforderungen an staatliche Finanzierung die gleiche Struktur bezüglich der Beziehung zwischen Staatsausgaben und Wirtschaftswachstum unterstellt wird. Eine Ausgabenhöhe, die in den skandinavischen Staaten mit ihrem stark ausgebauten Wohlfahrtsstaat von der Bevölkerung akzeptiert wird und damit bestenfalls geringe negative Auswirkungen auf das Wirtschaftswachstum haben sollte, dürfte in den angelsächsischen Staaten kaum akzeptiert werden und damit auch das Wirtschaftswachstum erheblich beeinträchtigen. Um diese institutionellen Differenzen, die auch durch feste Effekte in den entsprechenden Panelregressionen kaum korrekt erfasst werden dürften, auszuschalten, hat Schaltegger (2004), eine Untersuchung für die Schweizer Kantone durchgeführt. ${ }^{17}$

Schaltegger (2004) schätzt Beziehung (1) zunächst mit Hilfe der gewöhnlichen KleinstQuadrate-Methode mit einem Panel für die Schweizer Kantone für den Zeitraum von 1981 bis 2001. Neben der verzögerten Wachstumsrate und der Staats- bzw. der Steuerquote berücksichtigt er als zusätzliche erklärende Variablen die privaten Investitionen, die Erwerbsquote, die Urbanisierung, die Größe der Bevölkerung, die Anteile der Bevölkerung unter 19 und über 65 Jahren, eine Hilfsvariable für den deutschen Sprachraum sowie die Arbeitslosen- und die Maturandenquote. Dabei kommt er zu dem Ergebnis, dass die Staatsquote einen hoch signifikanten, die Steuerquote bestenfalls einen marginal signifikanten negativen Einfluss auf das Wirtschaftswachstum der Schweiz hatte. Dies zeigen die in Tabelle 1 nachgeschätzten Ergebnisse seiner beiden wohl wichtigsten Gleichungen, ${ }^{18}$ wobei die Steuervariable jedoch nicht einmal marginal signifikant ist; ihre Signifikanz ist von jedem konventionellen Signifikanzniveau weit entfernt. Der Grund für die hier gegenüber den Angaben bei Schaltegger (2004, S. 5) deutlich niedrigeren t-Statistiken liegt darin, dass wir die geschätzten Varianzen der Koeffizienten mit Hilfe des Newey-West-Verfahrens um mögliche Autokorrelation und Heteroskedastie korrigiert haben. Dies ist deshalb erforderlich, weil die ebenfalls in Tabelle 1 wiedergegebene Ljung-Box Q-Statistik zeigt, dass die geschätzten Residuen erhebliche positive Autokorrelation erster Ordnung aufweisen, und die Jarque-Bera-Teststatistik zeigt, dass die Nullhypothese der Normalverteilung der Residuen auf jedem konventionellen Signifikanzniveau verworfen werden muss. Daher sind die traditionellen Standardfehler der geschätzten Parameter nach unten verzerrt, und die t-Statistiken zeigen möglicherweise Signifikanz an, obwohl diese gar nicht existiert.

17. Diese Untersuchung wurde ausgelöst durch eine Anfrage des Walliser Nationalratsabgeordneten Jean-Noël Rey an den Schweizer Bundesrat. Christoph Schaltegger war damals wirtschaftspolitischer Berater in der Eidgenössischen Steuerverwaltung in Bern und hat diese Studie in dieser Funktion erstellt. In der später veröffentlichten Arbeit zusammen mit Benno Torgler (Schaltegger und Torgler (2006)) wurde die Untersuchung ausgeweitet, ohne dass die grundsätzlichen Ergebnisse sich änderten.

18. Die Daten wurden mir freundlicherweise von Christoph Schaltegger zur Verfügung gestellt. 


\begin{tabular}{|c|c|c|c|c|}
\hline \multicolumn{5}{|c|}{$\begin{array}{l}\text { Tabelle 1: Wachstumsraten des realen Bruttoinlandsprodukts pro Kopf, } \\
1980-2001,546 \text { Beobachtungen }\end{array}$} \\
\hline Modell & (1) & (2) & (3) & (5) \\
\hline Verzögertes BIP & $\begin{array}{l}-0.142 * * * \\
(4.89)\end{array}$ & $\begin{array}{l}-0.129 * * * \\
(5.08)\end{array}$ & $\begin{array}{l}-0.137 * * * \\
(5.69)\end{array}$ & $\begin{array}{l}-0.122 * * * \\
(4.97)\end{array}$ \\
\hline Staatsquote & $\begin{array}{l}-0.064 * * \\
(3.11)\end{array}$ & & & \\
\hline Steuerquote & & $\begin{array}{l}-0.010 \\
(0.78)\end{array}$ & & \\
\hline Staatsausgaben pro Kopf & & & $\begin{array}{l}0.036(*) \\
(1.95)\end{array}$ & \\
\hline Steuereinnahmen pro Kopf & & & & $\begin{array}{l}0.011\left(^{*}\right) \\
(1.86)\end{array}$ \\
\hline Private Investitionen & $\begin{array}{c}0.003 \\
(0.46)\end{array}$ & $\begin{array}{l}-0.010 \\
(0.98)\end{array}$ & $\begin{array}{l}-0.017^{*} \\
(2.02)\end{array}$ & $\begin{array}{l}-0.010 \\
(1.57)\end{array}$ \\
\hline Erwerbsquote & $\begin{array}{c}0.046 \\
(1.59)\end{array}$ & $\begin{array}{r}0.029 \\
(0.98)\end{array}$ & $\begin{array}{c}0.014 \\
(0.50)\end{array}$ & $\begin{array}{c}0.024 \\
(0.82)\end{array}$ \\
\hline Urbanisierung & $\begin{array}{c}0.012 \\
(0.76)\end{array}$ & $\begin{array}{l}0.023 \\
(1.42)\end{array}$ & $\begin{array}{l}0.027(*) \\
(1.66)\end{array}$ & $\begin{array}{l}0.023 \\
(1.42)\end{array}$ \\
\hline Bevölkerung & $\begin{array}{l}-0.109 * * \\
(2.70)\end{array}$ & $\begin{array}{l}-0.109 * * \\
(2.70)\end{array}$ & $\begin{array}{l}-0.112^{* * *} \\
(2.71)\end{array}$ & $\begin{array}{l}-0.118 * * \\
(2.87)\end{array}$ \\
\hline $\begin{array}{l}\text { Anteil der Bevölkerung } \\
\text { unter } 19 \text { Jahren }\end{array}$ & $\begin{array}{l}0.102 \\
(1.43)\end{array}$ & $\begin{array}{l}0.118\left(^{*}\right) \\
(1.71)\end{array}$ & $\begin{array}{l}0.107 \\
(1.52)\end{array}$ & $\begin{array}{l}0.093 \\
(1.33)\end{array}$ \\
\hline $\begin{array}{l}\text { Anteil der Bevölkerung } \\
\text { über } 65 \text { Jahren }\end{array}$ & $\begin{array}{l}0.129(*) \\
(1.93)\end{array}$ & $\begin{array}{l}0.187^{*} \\
(2.46)\end{array}$ & $\begin{array}{l}0.218^{* * *} \\
(2.97)\end{array}$ & $\begin{array}{l}0.93 * * \\
(2.72)\end{array}$ \\
\hline $\begin{array}{l}\text { Hilfsvariable für den deut- } \\
\text { schen Sprachraum }\end{array}$ & $\begin{array}{l}-0.031 \\
(0.96)\end{array}$ & $\begin{array}{l}-0.019 \\
(0.59)\end{array}$ & $\begin{array}{l}-0.009 \\
(0.28)\end{array}$ & $\begin{array}{l}-0.019 \\
(0.62)\end{array}$ \\
\hline Maturandenquote & $\begin{array}{l}-0.021 \\
(0.83)\end{array}$ & $\begin{array}{l}-0.021 \\
(0.83)\end{array}$ & $\begin{array}{l}-0.032 \\
(1.22)\end{array}$ & $\begin{array}{l}-0.027 \\
(1.03)\end{array}$ \\
\hline Arbeitslosenquote & $\begin{array}{l}-0.002 * * \\
(3.31)\end{array}$ & $\begin{array}{l}-0.002 * * \\
(3.25)\end{array}$ & $\begin{array}{l}-0.002 * * \\
(3.07)\end{array}$ & $\begin{array}{l}-0.002 * * \\
(3.04)\end{array}$ \\
\hline$\overline{\mathrm{R}}^{2}$ & 0.592 & 0.576 & 0.579 & 0.577 \\
\hline Standardfehler & 0.007 & 0.007 & 0.007 & 0.007 \\
\hline $\mathrm{Q}(1)$ & $7.077 * *$ & $6.630 * *$ & $6.090^{*}$ & $4.411^{*}$ \\
\hline J.-B. & $710.773 * * *$ & $921.881 * * *$ & $1052.154 * * *$ & $1077.998 * * *$ \\
\hline \multicolumn{5}{|c|}{$\begin{array}{l}\text { Die Zahlen in Klammern sind die absoluten Werte der geschätzten t-Statistiken, wo- } \\
\text { bei die Standardfehler nach Newey-West korrigiert wurden. Q(1) ist der Wert der } \\
\text { Ljung-Box Q-Statistik mir einem Freiheitsgrad. J.-B. ist der Wert der Jarque-Bera } \\
\text { Teststatistik zur Überprüfung der geschätzten Residuen auf Normalverteilung. '***', } \\
\text { '**', '*' bzw. '(*)'geben an, dass die entsprechende Nullhypothese auf dem } 0.1,1 \text {, } \\
\text { 5, bzw. } 10 \text { Prozent Signifikanzniveau verworfen werden kann. }\end{array}$} \\
\hline
\end{tabular}


Die unterschiedlichen Ergebnisse für die Staats- und die Steuerquote deuten auf die oben besprochene Umkehrkausalität hin. Will man diesen Effekt ausschalten, kann man anstelle der Staatsquote die Staatsausgaben (bzw. Steuern) pro Kopf als erklärende Variable verwenden. Dann ergeben sich aber sowohl für die Staatsausgaben als auch für die Steuern positive, auf dem 10 Prozent-Niveau signifikante Effekte; eine Ausdehnung der Staatstätigkeit scheint jetzt das Wirtschaftswachstum eher zu verstärken. Auch hier kann man jedoch argumentieren, dass Umkehrkausalität vorliegt: Kantone mit hoher Wirtschaftskraft haben nicht nur höhere Steuereinnahmen pro Kopf, sondern können sich auch leisten, den Bürgern mehr Leistungen zur Verfügung zu stellen.

Um den diese Ergebnisse erzeugenden Mechanismus zu sehen, macht es Sinn, vom traditionellen Modell zur Erklärung der Staatsausgaben in Abhängigkeit vom Einkommen auszugehen, d.h. von

$$
\mathrm{G}=\alpha+\beta \mathrm{Y},
$$

wobei G die Staatsausgaben und Y das Volkseinkommen sind. Generell angenommen werden kann, dass zwischen Staatsausgaben und Volkseinkommen eine positive Beziehung besteht, d.h. dass

$$
\frac{\partial \mathrm{G}}{\partial \mathrm{Y}}=\beta>0
$$

gilt. Für das Verhältnis zwischen Staatsquote und Volkseinkommen gilt jedoch

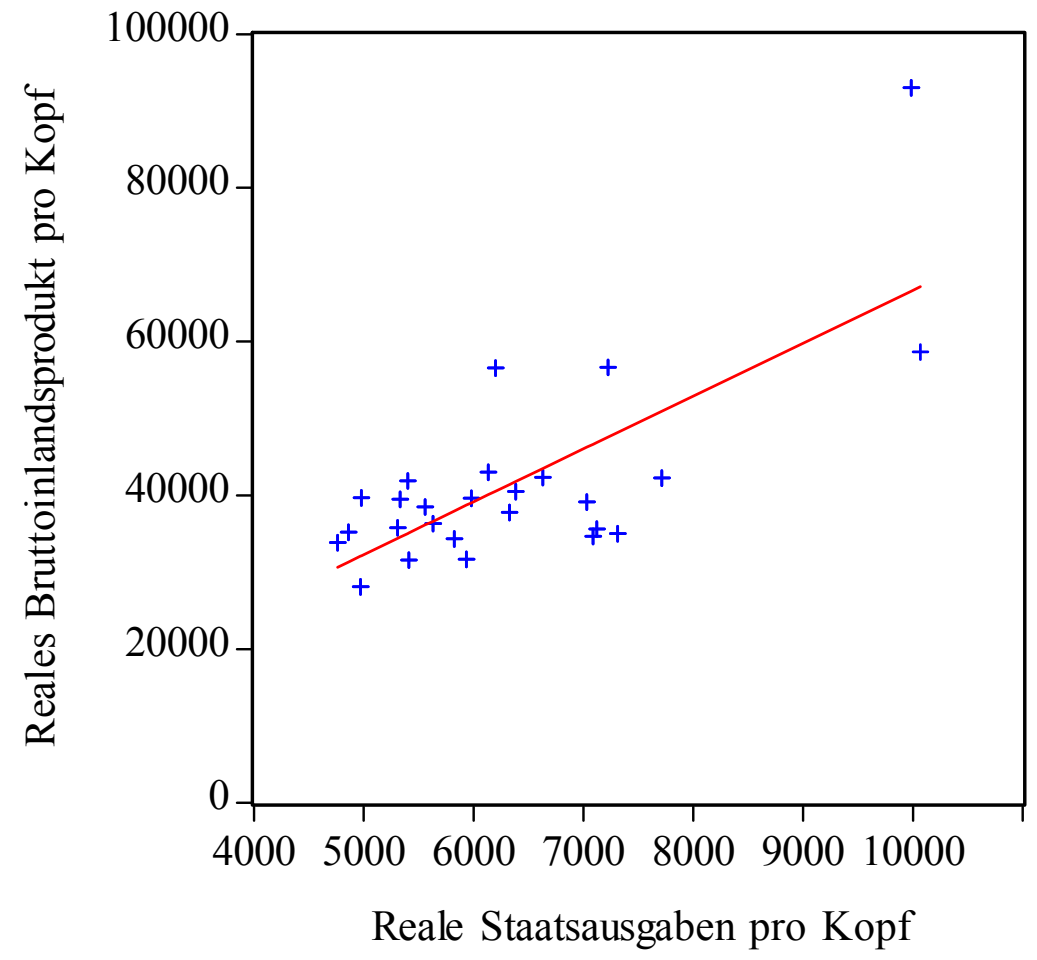

Abbildung 1: Zusammenhang zwischen Bruttoinlandsprodukt und Staatsausgaben Durchschnittswerte, 1981 - 2001 


$$
\frac{\partial \frac{G}{Y}}{\partial Y}=-\frac{\alpha}{Y^{2}}<0 \text { für } \alpha>0,
$$

d.h. wir finden eine negative Beziehung.

Um diesen Zusammenhang zu verdeutlichen, haben wir für die Ausgabenquote, die realen Ausgaben pro Kopf sowie die reale Wachstumsrate Durchschnittswerte für die einzelnen Kantone über den Zeitraum von 1981 bis 2001 gebildet. Mit diesen Daten ergibt eine Schätzung von Beziehung (2)

$$
\mathrm{G}_{\mathrm{i}}=\underset{(6.01)}{3.152}+\underset{(5.85)}{0.077} \mathrm{Y}_{\mathrm{i}},
$$

wobei die Staatsausgaben $(\mathrm{G})$ und Bruttoinlandsprodukt (Y) hier in 1000 Franken pro Kopf gemessen werden. Die entsprechende Beziehung ist in Abbildung 1 dargestellt. ${ }^{19}$ Die Korrelation zwischen beiden Variablen beträgt $0.729 .^{20}$

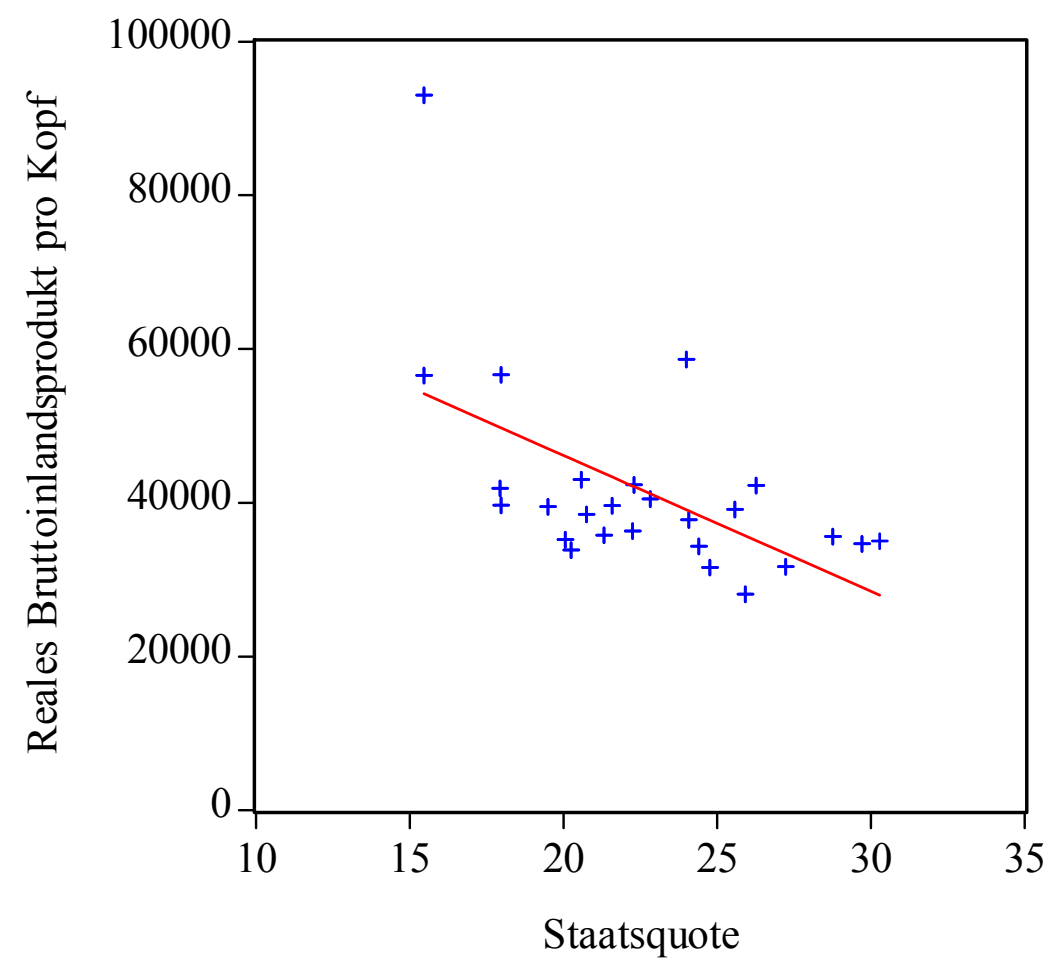

Abbildung 2: Zusammenhang zwischen Bruttoinlandsprodukt und Staatsquote Durchschnittswerte, 1981 - 2001

19. Die Berechnung der t-Statistiken wurde wieder mit Heteroskedastie-konsistenten Varianzen durchgeführt.

20. Eliminiert man den ,Ausreißer` Basel-Stadt, der (aufgrund seiner Struktur) mit Abstand das höchste Bruttoinlandsprodukt pro Kopf hat, beträgt die Korrelation immer noch 0.584. - Hier geht es nicht um einen statistischen Test einer kausalen Interpretation; vielmehr soll ein Effekt aufgezeigt werden, der sich auch in den oben aufgezeigten Schätzungen niederschlägt. 
Für das Verhältnis zwischen Staatsquote und Volkseinkommen erhalten wir jedoch folgende Schätzung:

$$
\mathrm{G}_{\mathrm{i}} / \mathrm{Y}_{\mathrm{i}}=\underset{(6.01)}{0.206-} \begin{gathered}
0.0116 \mathrm{Y}_{\mathrm{i}} \\
(5.85)
\end{gathered}
$$

d.h. wir finden jene negative Beziehung zwischen Staatsquote und Bruttoinlandsprodukt pro Kopf, die auch in Abbildung 2 erkennbar ist. Die Korrelation zwischen beiden Variablen beträgt $-0.557 . .^{21}$

Den gleichen Effekt erhalten wir, wenn wir anstelle von Beziehung (2) das multiplikative Modell

$$
\mathrm{G}=\alpha \cdot \mathrm{Y}^{\beta}
$$

verwenden, mit $\alpha>0$ und $0<\beta<1$. Durch Logarithmierung erhalten wir

$$
\mathrm{g}=\alpha^{\prime}+\beta \cdot \mathrm{y}
$$

mit $\alpha^{\prime}=\log (\alpha)$. Hier ergibt sich zwischen Staatsausgaben und Bruttoinlandsprodukt eine Korrelation von 0.693 und zwischen Staatsquote und Bruttoinlandsprodukt eine Korrelation von -0.628 .

Damit dies auch zu einem Zusammenhang zwischen der Wachstumsrate der Wirtschaft auf der einen Seite und den Staatsausgaben und Steuereinnahmen pro Kopf auf der anderen Seite führt, müssen jene Kantone, die ein hohes Bruttoinlandsprodukt pro Kopf aufweisen, auch stärker wachsen. Tatsächlich beobachten wir für den Zeitraum von 1981 bis 2001 eine Korrelation von 0.510 zwischen der durchschnittlichen Wachstumsrate und dem durchschnittlichen Bruttoinlandsprodukt pro Kopf in den Kantonen. Eine derartige positive Korrelation ist jedoch keineswegs selbstverständlich, zumindest wenn man die neoklassische Wachstumstheorie zugrunde legt: Die ,Catch Up-Hypothese' dieser Theorie behauptet eine Konvergenz der Entwicklung, weshalb man eher den umgekehrten Zusammenhang erwarten sollte. Tatsächlich haben die verzögerten Werte des Bruttoinlandsprodukts in allen von Schaltegger (2004, S. 5) vorgestellten Schätzungen signifikant negative Koeffizienten, was für eine solche Konvergenz spricht. Gleiches gilt auch für unsere Schätzungen mit den Staatsausgaben bzw. Steuern pro Kopf. Dabei stellt sich freilich die Frage, wie schnell sich eine solche Konvergenz vollzieht. Die geschätzten Koeffizienten implizieren, dass die mittlere Anpassungsdauer etwa 7 bis 12 Jahre beträgt. Damit ist ein positiver Zusammenhang zwischen der Höhe des Bruttoinlandsprodukts pro Kopf und der Rate des wirtschaftlichen Wachstums auch über längere Zeit vereinbar. Dies führt zu dem in Abbildung 3 gezeigten Zusammenhang zwischen den realen Staatsausgaben pro Kopf und dem Wirtschaftswachstum; der Zusammenhang ist zwar nicht so stark wie jener zwischen den realen Staatsausgaben und dem Bruttoinlandsprodukt pro Kopf, aber immer noch positiv; der Korrelationskoeffizient beträgt 0.306. Abbildung 4 zeigt den Zusammenhang zwischen der Ausgabenquote und dem Wirtschaftswachstum; hier ist der Zusammenhang mit -0.266 wie erwartet negativ.

21. Eliminiert man auch hier den Wert von Basel-Stadt, beträgt die Korrelation noch -0.490 . 


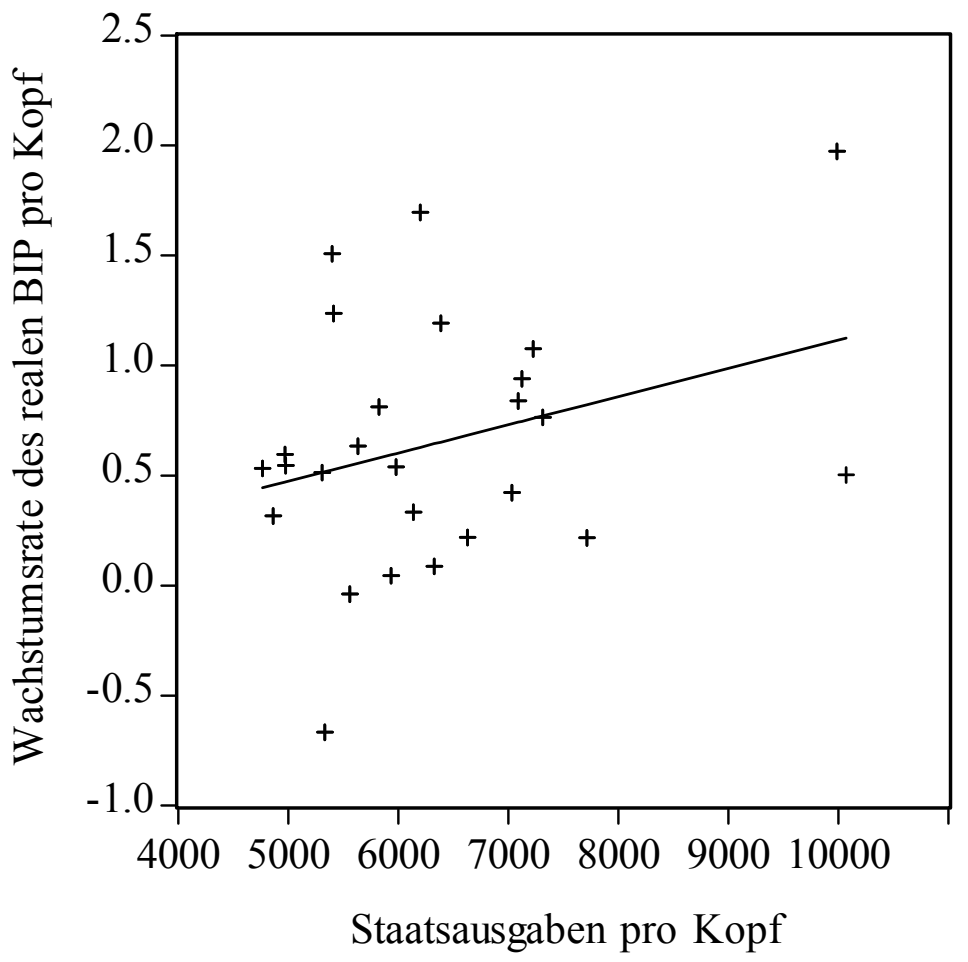

Abbildung 3: Zusammenhang zwischen Wirtschaftswachstum und Staatsausgaben pro Kopf Durchschnittswerte, 1981 - 2001

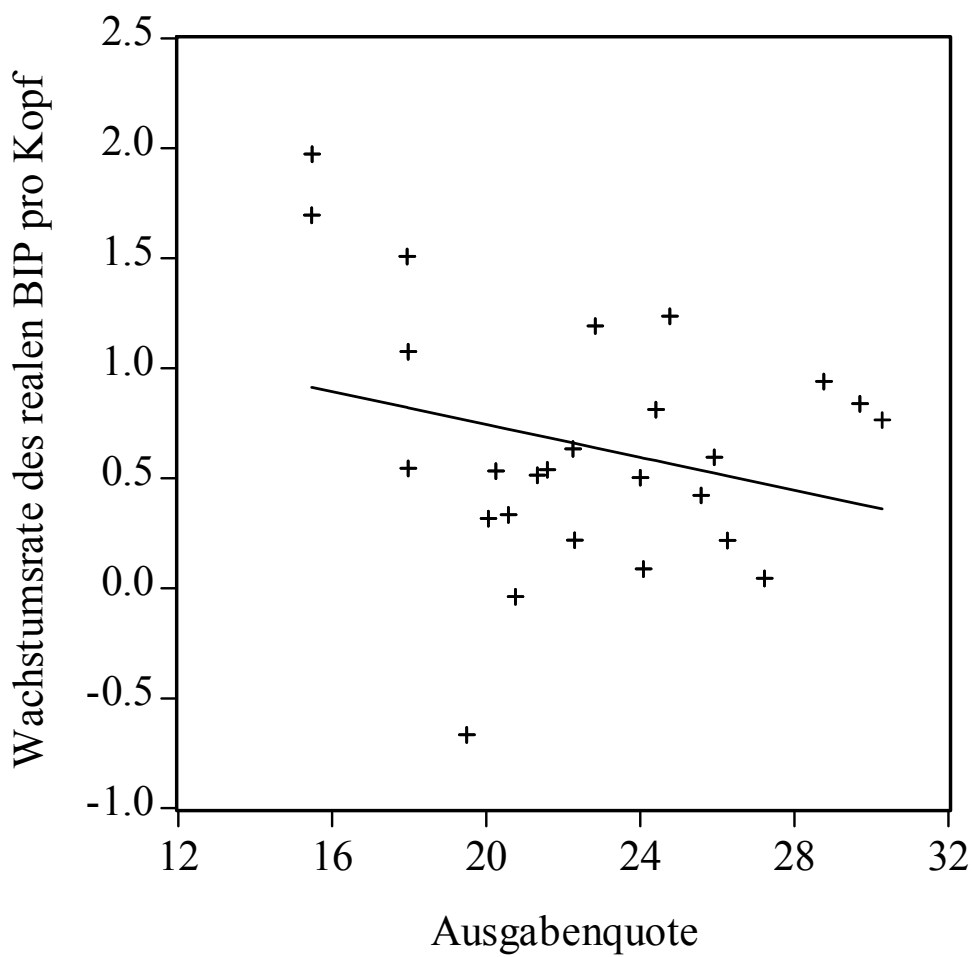

Abbildung 4: Zusammenhang zwischen Wirtschaftswachstum und Staatsquote Durchschnittswerte, 1981 - 2001 
Gerade angesichts der positiven Korrelation zwischen dem Bruttoinlandsprodukt pro Kopf und seiner Wachstumsrate dürfte sich der wesentliche Effekt des gemessenen Zusammenhangs zwischen der wirtschaftlichen Entwicklung und den Staatsausgaben vor allem aus der föderalen Struktur der Schweiz ergeben. Kantone mit hoher Wirtschaftskraft und hohem Wirtschaftswachstum können ihren Bürgen relativ viele Leistungen zukommen lassen und haben dennoch vergleichsweise geringe Steuersätze. Damit die ärmeren Kantone für ihre Bürger die gleichen Leistungen erbringen können, müssen deren Steuersätze sehr viel höher sein, was eine höhere Staatsquote mit sich bringt. Nun erbringen nicht alle Kantone die gleichen Leistungen, aber ein gewisses Mindestmaß an Leistungen, z.B. in den Bereichen Schule und Erziehung, Verkehrswesen oder allgemeine Verwaltung, muss überall erbracht werden. Zwar werden die Ausgaben pro Kopf in den ärmeren Kantonen geringer sein, nicht zuletzt weil ärmere Kantone ihren Beamten und Angestellten im Allgemeinen geringere Löhne zahlen als Kantone mit hoher Wirtschaftskraft. Dies ist jedoch nicht hinreichend, um diesen Kantonen ähnlich niedrige Steuersätze zu ermöglichen als den wohlhabenderen Kantonen. Alles spricht somit dafür, dass sich die gefundenen negativen Beziehungen zwischen der wirtschaftlichen Entwicklung und der Staatsquote aus der Umkehrkausalität ergeben; sie lassen keine Schlüsse darüber zu, wie sich eine bestimmte Höhe der Staatsquote auf die wirtschaftliche Entwicklung auswirkt. Für unsere Diskussion ist jedoch wichtig, dass dieses Beispiel zeigt, wie mit den gleichen Daten ganz unterschiedliche Ergebnisse erzielt werden können, die zu entgegengesetzten politischen Schlussfolgerungen führen können, ohne dass man Manipulation unterstellen müsste. ${ }^{22}$

\section{Die Abschreckungswirkung der Todesstrafe}

Ob die Todesstrafe abschreckend wirkt, ist eigentlich keine im engeren Sinne ökonomische Fragestellung; sie gehört in das Gebiet der (empirischen) Rechtswissenschaft bzw. der Kriminologie. Andererseits ist es ein nahezu ideales Beispiel für die ökonomische Analyse des Rechts, da mit der Todesstrafe ein radikaler Anreiz gesetzt wird, der ein bestimmtes, nicht erwünschtes Verhalten unterbinden soll. $\mathrm{Ob}$ und inwieweit die Todesstrafe abschreckend wirkt, wird schon sehr lange diskutiert. Falls es überhaupt ein Argument für die Beibehaltung der Todesstrafe gibt, welches heute noch auf breite Zustimmung stoßen könnte, dürfte dies der Nachweis einer erheblichen Abschreckungswirkung sein. Tatsächlich überzeugt dieses Argument in den Vereinigten Staaten heute (noch) eine Mehrheit der Bevölkerung.

Die erste Arbeit, in welcher von einem Ökonomen ökonometrische Schätz- und Testverfahren eingesetzt wurden, um eine Abschreckungswirkung aufzuzeigen, stammt von Ehrlich (1975). Mit einer Zeitreihe von Jahresdaten von 1933 bis 1967 für die Vereinigten Staaten kam er zu dem Ergebnis, dass in diesem Zeitraum jede zusätzliche Hinrichtung dazu führte, dass ein bis acht weniger Morde geschahen. ${ }^{23}$ Unabhängig von diesem Ergebnis änderte die Arbeit von Ehrlich (1975) die Situation insofern, als danach zunehmend modernere ökonometrische

22. Eine ausführlichere Diskussion findet sich in Kirchgässner (2004).

23. Siehe hierzu Ehrlich (1975, S. 398). 
Schätz- und Testverfahren eingesetzt wurden, um die gewonnenen Aussagen abzusichern. An dieser Diskussion haben sich nicht nur Ökonomen beteiligt, sondern auch Juristen, Soziologen und Kriminologen. Dementsprechend ist die Literatur auch über die Zeitschriften dieser Disziplinen verstreut. Dabei stehen die anderen Wissenschaftler den Ökonomen in ihrer Methodenkenntnis heute in nichts mehr nach. Sie verwenden die gleichen Verfahren, arbeiten häufig auch mit den gleichen Daten und kommen doch zu deutlich abweichenden Ergebnissen: ${ }^{24}$ Während die meisten (amerikanischen) Ökonomen an die Gültigkeit der Abschreckungshypothese glauben und entsprechende Ergebnisse vorlegen, haben die meisten, wenn auch nicht alle Wissenschaftler anderer Fakultäten, insbesondere die Juristen, Zweifel an der Gültigkeit dieser Hypothese und demonstrieren die Fragilität der von den Ökonomen vorgelegten Ergebnisse. Da beide Seiten die gleichen fortgeschrittenen statistischen Verfahren anwenden, drehen sich die Diskussionen auch um methodische Probleme, z.B. um die Qualität der bei Instrumentenschätzern verwendeten Instrumente.

Angesichts dieser Situation kann man den Eindruck gewinnen, die ökonomische Analyse der Kriminalität sowie insbesondere die Diskussion über die Abschreckungswirkung der Todesstrafe sei nichts anderes als ein Tummelplatz für Ideologen. Dass politische Einstellungen einen Einfluss auf die vorgelegten Ergebnisse zur Abschreckungswirkung der Todesstrafe haben könnten, wurde bereits von McManus (1985) aufgezeigt. Dies muss nicht bedeuten, dass die Autoren ihre Ergebnisse bewusst fälschen; es kann schlicht das Ergebnis selektiver Wahrnehmung sein: Wenn unterschiedliche Ergebnisse möglich sind, wählen die Autoren jene aus, von denen sie von vornherein überzeugt sind, und sie suchen nach starken Argumenten, welche diese Ergebnisse unterstützen.

Wie man zu so unterschiedlichen Ergebnissen kommen kann, kann mit einer Meta-Studie untersucht werden. Solche Studien können zwei Ziele haben: Man kann zum einen versuchen, die gesamte empirische Evidenz zusammenzufassen, die z.B. zur Schätzung einer bestimmten Nachfrageelastizität verfügbar ist, um zuverlässigere Information über den ,wahren ' Wert dieser Größe zu erhalten. Dies kann dann Sinn machen, wenn die verschiedenen Autoren den gleichen oder zumindest verwandte theoretische Ansätze, aber z.B. Daten unterschiedlicher Länder oder Zeitperioden verwenden. Das zweite Ziel einer solchen Untersuchung kann sein herauszufinden, was die Ursachen für die unterschiedlichen Ergebnisse sind. Dies können z.B. unterschiedliche Zeitperioden, aber auch verschiedene Schätzverfahren sein. Da es im Falle der Abschreckungswirkung der Todesstrafe zwei klar abgrenzbare Lager von Autoren zu geben scheint, die zu unterschiedlichen Ergebnissen kommen, ist hier nur die zweite Fragestellung angebracht. Im Folgenden sollen deshalb die Ergebnisse einer solchen MetaAnalyse vorgestellt werden. ${ }^{25}$

24. Siehe hierzu Kirchgässner (2011a).

25. Zur genaueren Analyse siehe Gerritzen und Kirchgässner (2012). - Bisher liegen drei Metaanalysen vor: Yang und Lester (2008), Dölling et al. (2009) sowie Herman (2010). Yang und Lester (2008) fassen die Ergebnisse der von ihnen untersuchten Studien im Wesentlichen in Tabellen zusammen und bilden dann Durchschnittswerte der geschätzten Effekte. Dies führt sie zur der Schlussfolgerung, dass zwar die Mehrheit der Studien auf einen Abschreckungseffekt hinweist, dass die Ergebnisse aber wesentlich durch die 
Beginnend mit der Arbeit von Ehrlich (1975), haben wir versucht, sämtliche Originalarbeiten zu berücksichtigen, die eine Antwort auf diese Frage zu geben versuchten. Von den insgesamt 101 von uns erfassten Arbeiten kommen 87 zu eindeutigen Aussagen: 34 Arbeiten kommen zu dem Ergebnis, dass ein signifikanter Abschreckungseffekt besteht, welcher die Verhängung der Todesstrafe rechtfertigen soll, während dies in 53 Arbeiten bestritten wird. ${ }^{26}$ Ein Überblick über diese Arbeiten, wie er z.B. in Kirchgässner (2011) über die Arbeiten des vergangenen Jahrzehnts gegeben wurde, lässt vermuten, dass die Ergebnisse wesentlich davon abhängen, ob Ökonomen oder Nicht-Ökonomen die Schätzungen durchgeführt haben. Dabei können selbstverständlich auch andere Faktoren eine Rolle spielen wie z.B. die Art der verwendeten Daten oder Schätzverfahren. Weiter könnte es eine Rolle gespielt haben, ob in den Schätzgleichungen, die ja die Zahl der Morde in einem Jahr und einer bestimmten Region erklären sollten, die wirtschaftliche Entwicklung als zusätzlicher Erklärungsfaktor berücksichtigt wurde. Schließlich könnte sein, dass es in ökonomischen Zeitschriften leichter ist, Ergebnisse zu publizieren, die einen Abschreckungseffekt bestätigen, als in nicht-ökonomischen Zeitschriften, oder auch dass dies in wissenschaftlichen Zeitschriften schwieriger ist als in Zeitungen. In diesem Fall würde es sich zwar um Umkehrkausalität handeln, aber auch dies sollte in einer solchen Schätzgleichung deutlich werden.

Als Startpunkt wurde folgende Gleichung geschätzt:

(3) $\mathrm{DET}=\mathrm{f}(\mathrm{AECON}, \mathrm{ANECON}$, ECONJ, NECONJ, TS, CS, OLS, INST, WLS, US, JAHR, ALQ, Y)

mit:

DET Die Arbeit findet einen signifikanten Abschreckungseffekt.

AECON Der (ein) Autor ist ein Ökonom.

ANECON Der (ein) Autor ist kein Ökonom.

ECONJ Das Papier wurde in einer wirtschaftswissenschaftlichen Zeitschrift veröffentlicht.

NECONJ Das Papier wurde in einer wissenschaftlichen, nicht wirtschaftswissensschaftlichen Zeitschrift veröffentlicht.

TS Es werden Zeitreihendaten verwendet.

CS Es werden Querschnittsdaten verwendet.

OLS Das gewöhnliche Kleinst-Quadrate-Verfahren (OLS) wird verwendet.

INST Es werden fortgeschrittene Schätzverfahren (Instrumentenschätzer, GMM) eingesetzt.

verwendeten Daten bestimmt sind: Untersuchungen mit Zeitreihen- und Paneldaten sprechen eher für einen Abschreckungseffekt als reine Querschnittsanalysen. Im Gegensatz dazu finden Dölling et al. (2009), die allgemein die Abschreckungswirkung von Strafen untersuchen, keinen signifikanten Effekt bei der Todesstrafe. Hermann (2010) ist der einzige, der nach dem Einfluss der Fachrichtung der Forscher fragt. Er findet, dass diese den größten Einfluss auf die Ergebnisse hat.

26. Wir kommen zu einer anderen Klassifikation verschiedener Arbeiten als Yang und Lester (2008), da wir berücksichtigen, ob der geschätzte Abschreckungseffekt statistisch signifikant ist oder nicht, während sie allein das Vorzeichen betrachten. 
WLS Die Beobachtungen werden gewichtet.

US Daten aus den Vereinigten Staaten werden verwendet.

JAHR Jahr der Veröffentlichung.

ALQ In die Gleichung wird die Arbeitslosenquote aufgenommen.

Y In die Gleichung wird die Wachstumsrate des Einkommens aufgenommen.

Mit Ausnahme von JAHR handelt es sich um binäre Variablen.

In einer ersten Schätzung, die mit einem Probit-Ansatz durchgeführt wurde, wurden sowohl die Hilfsvariable für einen Ökonomen als Autor (AECON) als auch jene für einen NichtÖkonomen (ANECON) als Autor als erklärende Variable berücksichtigt, da es auch Arbeiten mit sowohl einem Ökonomen als auch einem Nicht-Ökonomen als Autor gibt. Da dies aber nur sehr wenige Arbeiten sind, sind diese beiden Variablen sehr hoch negativ korreliert, weshalb beide nicht signifikant waren. Gemeinsam sind sie jedoch extrem hoch signifikant, der Wert des Wald-Tests beträgt $\hat{\chi}^{2}(2)=15.34$, was bei zwei Freiheitsgraden noch auf einem Niveau von 0.05 Prozent signifikant ist. Im Folgenden haben wir deshalb nur noch die Variable für die Ökonomen als Autor in der Schätzgleichung berücksichtigt. Damit wurde diese Variable hoch signifikant. Gleichzeitig haben sich die Werte aller Informationskriterien verbessert.

Die Hilfsvariablen für Zeitreihen- (TS) und Querschnittsdaten (CS) wurden beide in die Schätzgleichung aufgenommen; bei Panels nehmen beide Variablen den Wert 1 an. Auch wurden drei Variablen für die verwendeten Verfahren eingeführt, OLS für das gewöhnliche Kleinst-Quadrate-Verfahren, WLS für gewichtete Schätzungen und INST für die Verwendung von Instrumentenschätzern sowie anderer fortgeschrittener Schätzverfahren. In der Restkategorie sind jene Untersuchungen enthalten, die mit einfachen Vergleichen arbeiten. Die wirtschaftliche Entwicklung wurde durch die Einkommensentwicklung (Y) und die Höhe der Arbeitslosigkeit (ALQ) abgebildet. Das Jahr der Veröffentlichung (JAHR) kann zeigen, ob in den Ergebnissen ein Trend vorhanden ist, falls z.B. die Todesstrafe im Zeitablauf an Akzeptanz gewonnen oder verloren hat. Die Variable für die Vereinigten Staaten schließlich soll zeigen, ob ein (signifikanter) Unterschied zwischen diesen und dem Vereinigten Königreich sowie Kanada besteht. Die Gleichung wurde mit dem Probit-Verfahren geschätzt, wobei die Standardfehler entsprechend den Autoren bzw. Autorengruppen geclustert wurden. Aus den 87 Beobachtungen wurden damit 51 Cluster gebildet.

Dies ergab folgende Schätzung: ${ }^{27}$

$$
\begin{aligned}
& \mathrm{DET}=-26.907+2.086 \mathrm{AECON}+0.658 \mathrm{ECONJ}+0.251 \mathrm{NECONJ} \\
& \begin{array}{llll}
(-0.65) & (3.38) & (0.95)
\end{array} \\
& -0.064 \mathrm{TS}-0.752 \mathrm{CS}-0.195 \mathrm{OLS}-0.456 \mathrm{INST}+0.365 \mathrm{WLS} \\
& \begin{array}{llll}
(-0.11) & (-1.52) \quad(-0.36) \quad(-1.09) \quad(0.90)
\end{array}
\end{aligned}
$$

27. Die Zahlen in Klammern sind die z-Werte der geschätzten Parameter. SER ist der Standardfehler der Residuen, Log LF der Logarithmus der Likelihoodfunktion, und H.-Q. der Wert des Hannan-Quinn Informationskriteriums. Die Schätzungen wurden mit STATA, Version 11.2 durchgeführt. 


$$
\begin{aligned}
& +0.908 \mathrm{US}+0.013 \mathrm{YEAR}+0.054 \mathrm{ALQ}-0.487 \mathrm{Y}+\hat{\varepsilon} \text {, } \\
& \text { (1.35) (0.60) (0.15) (-1.02) }
\end{aligned}
$$

Pseudo $\mathrm{R}^{2}=0.363, \quad$ SER $=0.491, \quad \log \mathrm{LF}=-37.067$, H.-Q. $=1.299$.

Geht man dann, wenn der geschätzte Wert für die Wahrscheinlichkeit, dass ein Abschreckungseffekt konstatiert wird, grösser als 0.5 ist, davon aus, dass in der betrachteten Arbeit ein Abschreckungseffekt behauptet wird, und dann, wenn dieser Wert kleiner als 0.5 ist, dass ein solcher Effekt bestritten wird, werden 27 Arbeiten derjenigen, die einen Abschreckungseffekt bejahen, sowie 43 Arbeiten derjenigen, die dies bestreiten, korrekt vorhergesagt. Die Trefferquote liegt damit bei 80.5 Prozent.

Mit Ausnahme der Variablen für die Profession des Autors hat keine der erklärenden Variablen einen signifikanten Einfluss. ${ }^{28}$ Dies kann freilich daran liegen, dass wir mit Ausnahme der Variablen für das Jahr jeweils mehrere Variablen in einer Gruppe haben, die, falls sie hoch miteinander korreliert sind, zwar nicht als einzelne, aber doch insgesamt einen signifikanten Einfluss ausüben könnten. Deshalb haben wir zunächst Likelihood-Verhältnistests für diese Gruppen durchgeführt. Dies ergab folgende Resultate:

a) Indikatoren für das Publikationsorgan (ECONJ, NECONJ):

$\log \operatorname{LF}=-37.708, \hat{\chi}^{2}(2)=1.10(\mathrm{p}=0.578)$.

b) Indikatoren für die verwendeten Daten (CS, TS):

$\log \operatorname{LF}=-38.325, \quad \hat{\chi}^{2}(2)=2.48(\mathrm{p}=0.289)$.

c) Indikatoren für die verwendeten Daten (OLS, WLS, INST):

$\log \operatorname{LF}=-37.746, \quad \hat{\chi}^{2}(3)=1.81(p=0.614)$.

d) Indikatoren für wirtschaftliche Entwicklung (ALQ, Y):

$\log \operatorname{LF}=-37.762, \quad \hat{\chi}^{2}(2)=1.03(\mathrm{p}=0.597)$.

In keinem dieser Fälle kann auf irgendeinem konventionellen Niveau ein signifikanter Einfluss festgestellt werden. Führt man einen gemeinsamen Test für alle diese Variablen (einschließlich USA und Jahr, die auch nicht signifikant waren) durch, so erhält man:

$$
\log \mathrm{LF}=-41.684, \hat{\chi}^{2}(11)=8.22(\mathrm{p}=0.694) .
$$

Damit können alle diese Variablen ausgeschlossen werden, womit wir zu folgender reduzierten Schätzung kommen:

$$
\begin{aligned}
\text { DET }= & -1.221+1.718 \text { AECON }+\hat{\varepsilon} \\
& (-2.65) \quad(3.30) \\
& \text { Pseudo } \mathrm{R}^{2}=0.284, \text { SER }=0.491, \quad \text { Log LF }=-41.684, \text { H.-Q. }=1.027
\end{aligned}
$$

Das Pseudo $\mathrm{R}^{2}$ ist jetzt zwar etwas geringer, aber der Standardfehler verändert sich kaum, und das Hannan-Quinn Kriterium verbessert sich erheblich. Insgesamt werden jetzt die Ergebnisse von 25 Arbeiten derjenigen, die einen Abschreckungseffekt behaupten, und 40 Arbeiten der-

28. Wir können damit das zentrale Ergebnis von Hermann (2010) unter Verwendung eines größeren Datensatzes bestätigen. 
jenigen, die dies bestreiten, korrekt vorhergesagt. Die Trefferquote verringert sich leicht auf 79.3 Prozent. Dabei ist die Profession (neben dem Absolutglied) die einzige erklärende Variable. Offensichtlich glauben die (amerikanischen) Ökonomen mit Mehrheit daran, dass die durch die Androhung der Todesstrafe bewirkten Anreize so groß sind, dass sie zumindest eine signifikant große Zahl an potentiellen Mördern von solchem Tun abhalten, während die Nichtökonomen mit großer Mehrheit davon ausgehen, dass Mordtaten zumindest vorwiegend aus anderen Motiven heraus geschehen, weshalb die Effekte dieser Anreize, falls sie überhaupt existieren, so gering sind, dass sie die Todesstrafe angesichts deren moralischer Problematik nicht rechtfertigen können. Dieser grundlegende Auffassungsunterschied führt dazu, dass sie selbst dann, wenn sie die gleichen Daten und Schätzverfahren verwenden, zu unterschiedlichen Ergebnissen kommen, indem sie z.B. geringfügig andere Spezifikationen für die Schätzgleichungen bzw. etwas andere Instrumente verwenden.

Für die Politikberatung ist dies insofern relevant, als ein Teil dieser Papiere nicht nur für die akademische Diskussion vorgesehen ist, sondern als Gutachten oder zumindest als Hintergrundmaterial für Anhörungen im amerikanischen Kongress verwendet wurde. ${ }^{29}$ Diese Anhörungen und damit auch die dort vorgetragenen Argumente können Auswirkungen auf die Gesetzgebung, aber auch auf die Rechtsprechung haben. Schließlich ist die Abschreckungswirkung das einzige Argument, welches in einem Diskurs über die Todesstrafe deren Anwendung überhaupt rechtfertigen könnte.

\section{$4 \quad$ Zum Prozess der Politikberatung}

Ökonomen haben sich bisher nicht sehr häufig mit dem Prozess der Politikberatung befasst. Wenn sie es getan haben, dann häufig, indem haben sie häufig darüber geklagt, dass die Politik ihren Ratschlägen nicht in ausreichendem Maße folge. ${ }^{30}$ Dabei war in den vergangenen Jahrzehnten wohl kaum eine Sozialwissenschaft politisch so erfolgreich wie die Ökonomie: Die Politiker hätten die großen Vorhaben der Deregulierung und Privatisierung wohl kaum jemals so umgesetzt, wenn nicht die überwiegende Mehrheit der Ökonominnen und Ökonomen ihnen permanent erklärt hätte, dass dies der allgemeinen Wohlfahrt diene.

Hinter den Klagen über die weitgehende Erfolglosigkeit der politischen Beratungstätigkeit von Wirtschaftswissenschaftlern steht das traditionelle, reichlich naive Verständnis vom Verhältnis zwischen Wissenschaft und Politik. Es geht von Idealbildern von Politikern wie Wissenschaftlern aus, die in der Realität nicht gegeben sind. Von der Politik wird erwartet, dass sie bestrebt ist, das Gemeinwohl zu fördern, und dabei die zuverlässigen Ergebnisse der Wissenschaft berücksichtigt. Von der Wissenschaft wird erwartet, dass sie diejenigen objektiven Informationen, welche die Politik benötigt, bereitwillig zur Verfügung stellt. ${ }^{31}$

29. Siehe z.B. Fagan (2005) oder Shepherd (2004).

30. Siehe hierzu z.B. Scheide (2005) oder Kruse (2010).

31 Zum Verhältnis von Wissenschaft und Politik siehe auch Kirchgässner (2007). 
Hinter diesem Verständnis steht eine ganze Reihe von Annahmen, die in aller Regel nicht oder bestenfalls zum Teil reflektiert werden:

(i) Die Regierung verhält sich wie ein wohlwollender Diktator. Ihr Handeln wird als Versuch der Maximierung einer Zielfunktion verstanden, welche die soziale Wohlfahrt abbildet. $^{32}$

(ii) Die Regierung ist für ihre Politik darauf angewiesen, dass sie von der Wissenschaft entsprechende Informationen erhält.

(iii) Die Wissenschaftler sind politisch neutral und nur der Wahrheit (bzw. der Wohlfahrt des Gemeinwesens) verpflichtet.

(iv) Die Wissenschaft gibt eindeutige Anweisungen.

Viele Ökonomen, aber auch andere (Sozial-)Wissenschaftler, haben von sich selbst als Wissenschaftler ein sehr hehres Bild. Sie glauben an dieses Bild auch dann, wenn sie Politikberatung betreiben: sie halten sich für objektiver und unabhängiger als andere Menschen (Nichtwissenschaftler). Sie glauben, dass sie aufgrund ihrer Wissenschaftlichkeit nicht nur über mehr Information verfügen und damit politische Fragen besser beurteilen können als andere Menschen, sondern dass sie auch eher dem Gemeinwohl dienen als jene. Sie billigen sich dabei eine moralische Position zu, welche sie (fast) niemand anderem zugestehen. ${ }^{33}$

Entsprechend dieser Perspektive besteht das Problem der Wissenschaft darin, den Politikern genau zu erklären und sie davon zu überzeugen, worin die gesellschaftliche Wohlfahrt besteht wie das Ziel ihrer Maximierung möglichst weitgehend erreicht werden kann. Da dies ganz offensichtlich bei Weitem nicht immer gelingt, befasst sich ein Großteil der Literatur zur Politikberatung, der von dieser Konzeption ausgeht, damit aufzuzeigen, wie man die Politiker möglicherweise auf den rechten Weg bringen könnte und wie dies in dem einen oder anderen Fall auch gelungen sein mag. ${ }^{34}$ Dies gilt auch für jene Beiträge, die sich mit dem Verhältnis zwischen empirischer Forschung und wirtschaftspolitischer Beratung befassen. ${ }^{35}$

Dass (demokratisch gewählte) Politiker sich generell wie wohlwollende Diktatoren verhalten, ist freilich eine sehr merkwürdige Vorstellung. Schließlich streben sie - gerade in einer Demokratie - ganz unterschiedliche Ziele an, die sich zum Teil diametral widersprechen. Auch wenn diese Annahme, dass die Regierung eine gesellschaftliche Wohlfahrtsfunktion maximiert, heute noch in vielen Modellen - z.B. der Theorie optimaler Besteuerung - anzutreffen ist, ist sie auch aus ökonomischer Perspektive kaum verständlich. Geht man als Ökonom davon aus, dass die Wirtschaftssubjekte vornehmlich ihre eigenen Interessen (selbstverständlich unter Nebenbedingungen) verfolgen, dann gibt es keinen Grund anzunehmen, dass die Politiker dies nicht auch tun. Beginnend mit den klassischen Arbeiten von Arrow (1951), Downs

32. Siehe hierzu explizit Tinbergen (1956, S. 50).

33. Siehe hierzu als besonders krasses Beispiel Hesse (1994, S. 18).

34. Siehe z.B. Frankel (2006).

35. Sieh hierzu z.B. Beiträge in Galler und Wagner (1998). 
(1957) und Buchanan und Tullock (1962) hat sich eine moderne Politische Ökonomie entwickelt, die das Verhalten politischer genauso wie jenes wirtschaftlicher Akteure mit Hilfe des ökonomischen Ansatzes untersucht und die heute in die moderne Volkswirtschaftslehre weitgehend integriert ist. Daraus ergab sich für die Theorie wirtschaftspolitischer Beratung eine neue Fragestellung. Während man angesichts der Beratungsresistenz vieler Politiker bisher fragte: „Warum folgte der Patient nicht den Anweisungen des Doktors?“" 36 stellte sich jetzt die Frage: „Warum sollte sich eine Regierung an die Ratschläge ihrer wissenschaftlichen Berater halten, es sei denn, diese Ratschläge liegen auch in ihrem eigenen Interesse?“ 37

Die Öffentlichkeit nimmt den Ökonomen ihr ideales Selbstbildnis kaum ab. Zwar genießen Professoren in der deutschen Bevölkerung nach wie vor ein vergleichsweise hohes Ansehen, wie sich z.B. aus der Allensbacher Berufsprestige Skala ergibt; insbesondere haben sie ein deutlich höheres Ansehen als die Politiker ${ }^{38}$ In der Finanz- und Wirtschaftskrise der vergangenen Jahre wurde die Reputation der Ökonomen jedoch stark beeinträchtigt. ${ }^{39}$ Aber auch wenn die in der Öffentlichkeit geäußerte Kritik in vielen Punkten überzogen sein mag, wird man doch zugestehen müssen, dass das Bild der Öffentlichkeit von den wissenschaftlichen Beratern eher realistisch ist als jenes hehre Bild, welches viele Ökonomen (und andere Wissenschaftler) von sich selbst haben. Schließlich verfassen sie z.B. Gutachten für unterschiedliche Interessengruppen, für die sie sich nicht selten recht gut bezahlen lassen. Und deren Ergebnisse dienen in aller Regel ihren Auftraggebern, wobei von verschiedenen Wissenschaftlern nicht selten sich widersprechende Gutachten vorgelegt werden. Da sie wissen, dass hier Konflikte mit ihrer Rolle als Wissenschaftler auftreten könnten, versuchen Gutachter zumindest teilweise ihre Einbindung in wirtschaftliche Interessen zu verschleiern. ${ }^{40}$ Nicht umsonst haben sowohl die American Economic Association als auch der Verein für Socialpolitik einen Ehrenkodex verabschiedet, der die Ökonomen z.B. verpflichtet, dann, wenn sie in einer der Zeitschriften dieser beiden Gesellschaften publizieren, mögliche Interessenkonflikte offen zu legen. $^{41}$

Dazu kommt ein systematisches Argument. Auch wenn sich viele Ökonomen und gerade auch solche, die sich der Public Choice-Schule zurechnen, dagegen sträuben: Man kann kaum allen anderen unterstellen, dass sie ihre eigenen Interessen verfolgen, und für sich selbst reklamieren, dass man allein an der gesellschaftlichen Wohlfahrt interessiert ist. Wenn man (bei Anwendung des ökonomischen Verhaltensmodells) davon ausgeht, dass alle Wirtschaftssubjekte und alle Politiker im Wesentlichen ihren eigenen Vorteil suchen, weshalb sollte man dann ausgerechnet die Ökonomen davon ausnehmen. Eine konsequente Abwendung des öko-

36. Siehe z.B. VanDoren (1989) oder, speziell für die Umweltpolitik, Hahn (1989).

37. Siehe hierzu z.B. Heine und Mause (2004) oder Jones und Cullis (1993).

38. Siehe hierzu die Allensbacher Berufsprestige-Skala 2011, in: Allensbacher Berichte 2/2011, April, (http://www.ifd-allensbach.de/uploads/tx_reportsndocs/prd_1102.pdf(15/11/12)).

39. Siehe hierzu Kirchgässner (2009, S. 437f.).

40. Siehe hierzu die Beispiele in Ferguson (2012, S. 240ff.)

41. Zum Kodex des Vereins für Socialpolitik siehe http://www.socialpolitik.org/inhalte/Ethikkodex_final.pdf $(14 / 11 / 12)$. 
nomischen Verhaltensmodells gestattet hier keine Ausnahme: Auch Ökonomen maximieren ihren Nutzen unter Nebenbedingungen. Sie unterscheiden sich darin nicht von anderen Menschen, sie sind nicht grundsätzlich anders, weder schlechter noch besser. ${ }^{42}$

Akzeptiert man dies, d.h. dass Ökonomen nicht per se bessere Menschen sind, dann stellen sich eine Reihe neuer Fragen. Es geht dann nicht mehr nur darum, warum der Patient nicht den Anweisungen seines Arztes folgte, sondern auch darum, warum der Doktor diese Anweisungen überhaupt gab.

Dies bedeutet aber auch, dass wir jenes Idealbild des Wissenschaftlers, welches Max Weber (1919, S. 84) uns vermittelte, indem er schrieb:

\section{„Persönlichkeit auf wissenschaftlichem Gebiet hat nur der, der rein der Sache dient.“}

nicht mit der Realität verwechseln dürfen. Und schon gar nicht sollten wir unterstellen, dass derart hehres Verhalten hinreichend oder gar notwendig für Objektivität in der Wissenschaft sei. So hat Karl Popper (1962, S. 112), der ein realistischeres Bild des Wissenschaftsbetrieb hatte, in seiner berühmten Diskussion mit Adorno (1962) im sogenannten ,Postitivismusstreit in der Deutschen Soziologie' ausgeführt:

„Es ist gänzlich verfehlt anzunehmen, dass die Objektivität der Wissenschaft von der Objektivität des Wissenschaftlers abhängt. ... Die Objektivität der Wissenschaft ist nicht eine individuelle Angelegenheit der verschiedenen Wissenschaftler, sondern eine soziale Angelegenheit ihrer gegenseitigen Kritik.“

Geht man davon aus, dass die politischen Berater (auch) eigene Interessen verfolgen, bedeutet dies nicht, dass sie deshalb auch korrupt sein müssten. Man kann dies zwar nicht ausschließen, und es gibt auch immer wieder Beispiele dafür. Aber genauso wenig wie alle Kaufleute versuchen, ihre jeweiligen Vertragspartner zu betrügen, muss man davon ausgehen, dass alle Wissenschaftler genauso wie alle Politiker ausschließlich auf ihr eigenes Wohl bedacht sind. Und wie bei den Kaufleuten sollte man freilich vorsichtig werden, wenn sie allzu fest behaupten, nur das Gemeinwohl im Auge zu haben. Denn was Adam Smith (1776, S. 371) über Kaufleute sagte,

„Alle, die jemals vorgaben, ihre Geschäfte dienten dem Wohl der Allgemeinheit, haben meines Wissens niemals etwas Gutes getan. Und tatsächlich ist es lediglich eine Heuchelei, die unter Kaufleuten nicht weit verbreitet ist, und es genügen schon wenige Worte, um sie davon abzubringen."

dürfte auch in Bezug auf Wissenschaftler und insbesondere auf Politikberater bedenkenswert sein.

Auch wenn man so vom Idealbild des Wissenschaftler abrückt und ihm ein realistischeres Bild entgegenstellt, sollte man die Existenz sich widersprechender wissenschaftlicher Gutachten nicht dadurch zu erklären suchen, dass die Beteiligten schlechte Menschen bzw. opportunistisch im Sinne von Williamson (1985, S. 54) sind, sondern man sollte - entsprechend des

42. Zum ökonomischen Verhaltensmodell siehe Kirchgässner (1991). 
ökonomischen Ansatzes - versuchen, dies als Ergebnisse rationalen Hadelns zu erklären, wobei die Akteure ihre eigenen Interessen verfolgen, ohne deshalb notwendigerweise juristisch oder auch nur moralisch fragwürdige Handlungen zu begehen. Auch wenn man opportunistisches Verhalten nie ganz ausschließen kann, spricht nichts dafür, dass sich wissenschaftliche Politikberater bei jeder sich bietenden Gelegenheit so verhalten.

Dies gilt auch, wenn Berater genau jene Ergebnisse vorlegen, welche die Auftraggeber von ihnen erwartet hatten. Es ist häufig der Fall, dass sowohl dem Auftraggeber als auch dem Auftragnehmer klar ist, welches Ergebnis herauskommen soll: Der Politiker weiß, welche Politik er verfolgen will, und er erwartet dafür vom Wissenschaftler entsprechende Unterstützung. Deshalb werden die Auftraggeber ihre Aufträge in aller Regel ganz bewusst an jene Gutachter vergeben, von denen sie sich entsprechende Ergebnisse erhoffen. Da die Gutachter diese Erwartungen kennen, ist davon auszugehen, dass die gewünschten Ergebnisse ihren eigenen ideologischen Vorstellungen zumindest nicht diametral entgegenstehen. Schließlich müssen sie sich damit bei Veröffentlichung der Gutachten auch identifizieren können.

Will man erreichen, dass mit diesen Gutachten (auch) Information vermittelt wird, ist vor allem darauf hinzuwirken, dass die Diskussion öffentlich stattfindet und dass die jeweiligen Grundlagen offengelegt werden. Dazu gehört auch, dass die Gutachten wissenschaftlichen Standards entsprechen und alle Quellen offengelegt werden. ${ }^{43}$ Bei empirischen Aussagen bedingt dies darüber hinaus die genaue Angabe der verwendeten Daten sowie die Offenlegung ihrer Quellen. ${ }^{44}$ Nur dann haben konkurrierende Interessengruppen sowie die Öffentlichkeit die Möglichkeit, die gemachten Aussagen kritisch zu hinterfragen. Dies aber ist notwendig, wenn es möglich sein soll, durch öffentliche Diskussion sachliche Information und politische Meinungsäußerung voneinander zu trennen. Beides hat selbstverständlich im politischen Diskurs seinen legitimen Platz, aber es sollte immer die Möglichkeit bestehen, beides voneinander zu trennen, so schwierig dies im Einzelfall auch sein mag.

Dass es, wie oben an Beispielen aufgezeigt wurde, möglich sein kann (und vermutlich häufig auch möglich ist), selbst mit den gleichen Daten und Verfahren bei seriöser Arbeit zu ganz unterschiedlichen Ergebnissen zu kommen, führt fast zwangsläufig dazu, dass die Wissenschaftler Ergebnisse veröffentlichen bzw. in Gutachten präsentieren werden, die mit ihren eigenen politischen Vorstellungen kompatibel sind. Wenn man von einer bestimmten Sache überzeugt ist und entsprechende empirische Ergebnisse findet, die zudem noch statistisch gut abgesichert erscheinen, hat auch der seriöse Wissenschaftler in aller Regel wenig Anlass, weiter nach Ergebnissen zu suchen, die dem widersprechen könnten. So können unterschiedliche politische Einstellungen infolge selektiver Wahrnehmung zu unterschiedlichen Ergebnissen

43. Dies war z.B. noch vor wenigen Jahren weder bei den Gutachten des Sachverständigenrats zur Begutachtung der gesamtwirtschaftlichen Entwicklung noch bei den Gutachten des Wissenschaftlichen Beirats des Bundesministeriums der Finanzen üblich. Sie glaubten selbst dort, wo sie Behauptungen über empirische Zusammenhänge aufstellten, ohne Quellenangaben auskommen zu können. Zur Kritik daran siehe Kirchgässner (1991).

44. Sollen die Daten vertraulich sein und z.B. wegen des Datenschutzes nicht an die Öffentlichkeit gelangen dürfen, kann man sie zumindest für Nachschätzungen zur Verfügung stellen, wobei die Vertraulichkeit selbstverständlich gewahrt bleiben muss. 
führen. Dies muss sich nicht auf Gutachten beschränken, sondern kann auch für rein wissenschaftliche Arbeiten gelten, und auch ohne dass hier finanzielle Anreize im Spiel sein müssen.

Dies besagt nicht, dass die Ergebnisse beliebig sind. Auch bei unterschiedlichen Stellungnahmen kann es eine kritische Diskussion geben, welche Fehler und Kunstgriffe einzelner Gutachter aufdeckt und so eine objektivere Beurteilung der Situation erlaubt. Die wesentliche Funktion einer solchen Diskussion ist es, mögliche Interessengebundenheiten der vorgebrachten Argumente aufzuzeigen, auch wenn diese mit der Rhetorik des Allgemeinwohls verbrämt werden. Eine solche aufklärerische Argumentation wird nicht das Wohlgefallen aller Betroffenen finden. Dennoch kann sie unabhängigen Dritten und insbesondere den politischen Entscheidungsträgern helfen, ,vernünftige‘ politische Entscheidungen zu treffen.

Man mag dies zugestehen aber einwenden, dass zum einen Wissenschaftler auch häufig nach Wahrheit streben und allein daran interessiert sind und dass zweitens Wissenschaftler bei Gutachten nicht immer das schreiben, was ihre Auftraggeber erwarten. Beides ist genauso wenig zu bestreiten wie die Beobachtung, dass Wissenschaftler häufig neben der Wahrheitssuche noch ganz andere Ziele verfolgen und dass sie bei Gutachten, welche von Interessengruppen vergeben werden, in der Regel das schreiben, was jene von ihnen erwarten.

Dabei ist jedoch zu berücksichtigen, dass auch für Wissenschaftler die Anreize davon abhängig sind, in welchem Umfeld sie sich bewegen. Für sie spielt im Allgemeinen die Reputation in der Wissenschaftlergemeinschaft eine wesentliche Rolle, und man wird diese wegen geringfügiger finanzieller Zuwendungen in aller Regel nicht aufs Spiel setzen wollen. Insofern wäre es gerade für eigeninteressierte Wissenschaftler nicht rational, sich für beliebiges Geld ,kaufen' zu lassen bzw. in Gutachten Ergebnisse zu produzieren, die sehr leicht zu widerlegen sind. Daher kann das Streben nach Objektivität durchaus dem Eigeninteresse entsprechen, auch wenn es anders erscheint oder dargestellt wird. Dennoch wird es (auch) bei Wissenschaftlern genuin nicht-eigennütziges (, altruistisches') Verhalten geben.

Bezüglich der Frage nach der Objektivität wissenschaftlicher Gutachten ist zusätzlich zu berücksichtigen, dass die beauftragten Wissenschaftler gerade dann, wenn sie Aufträge von Interessengruppen übernehmen, häufig weder anders können noch anders wollen, als das von ihren Auftraggebern angestrebte (politische) Ziel zu befürworten. Dies gilt auch dann, wenn sie sich nicht opportunistisch verhalten: Auch wenn alle Gutachter nur diejenigen Ziele verfolgen, von denen sie selbst überzeugt sind bzw. die sie selbst für (moralisch) gerechtfertigt halten, führt der Selektionsmechanismus zu einer solchen Zuordnung der Gutachter, dass die Zielvorstellungen von Auftraggebern und Gutachtern (weitgehend) übereinstimmen.

Dazu kommt, dass Wissenschaftler, soweit sie selbst politische Zielvorstellungen haben, dann, wenn sie als Gutachter tätig werden, auch von sich aus jene Auftraggeber suchen, die mit ihren politischen Zielen übereinstimmen. Als Staatsbürger haben sie - wie alle anderen das Recht, ihre eigenen Zielvorstellungen in den politischen Prozess einzubringen. Sie werden, soweit sie als Gutachter tätig sind, damit aber nur dann erfolgreich sein können, wenn ihre politischen Vorstellungen nicht zu stark von denjenigen ihrer Auftraggeber abweichen. Insofern wird die Interessenkonvergenz zwischen Auftraggeber und Auftragnehmer im Pro- 
zess der wissenschaftlichen Politikberatung von der Nachfrage- wie von der Angebotsseite befördert.

Da auch ,normale' wissenschaftliche Arbeiten im politischen Prozess sowie bei Gericht Beachtung finden und die Entscheidungsfindung beeinflussen können, genügt es nicht, nur bei Gutachten Transparenz herzustellen. Dies ist auch bei normalen wissenschaftlichen Arbeiten erforderlich. Es erfordert in erster Linie, dass mögliche Interessenkonflikte offen gelegt werden. Bei Gutachten ist klar erkennbar, wer der Auftraggeber ist. Wie die von Ferguson (2012, S. 240ff.) beschriebene Verquickung amerikanischer Ökonomen mit der Finanzindustrie zeigt, gibt es jedoch Fälle von Wissenschaftlern, die von wirtschaftlichen Interessengruppen in erheblichem Umfang finanziert werden und gleichzeitig in wissenschaftlichen Zeitschriften Arbeiten veröffentlichen, deren Resultate den Interessen ihrer außeruniversitären Financiers entgegenkommen. Dies muss nicht bedeuten, dass die Ergebnisse nicht korrekt sind. Möglicherweise kamen die Aufträge ja erst, nachdem erste Ergebnisse in dieser Richtung vorlagen, die völlig unabhängig von externen Auftraggebern erzielt wurden. Auch hier kann ein Prozess der Selbstselektion stattfinden. Gegenüber der Situation bei Gutachten gibt es jedoch zwei wesentliche Unterschiede. Zum einen werden an Publikationen in wissenschaftlichen Zeitschriften höhere Anforderungen an Originalität und Wissenschaftlichkeit gestellt als an Gutachten, die für die Politik oder für Gerichte erstellt werden. Zweitens sind bei Gutachten, soweit bekannt ist, wer sie beauftragt hat, in aller Regel keine weiteren Interessenkonflikte zu vermuten. Daher sollten auch bei wissenschaftlichen Arbeiten mögliche Interessenkonflikte aufgezeigt werden. In beiden Fällen aber sollten die verwendeten Daten und wesentliche Nicht-Standardprogramme zu Nachschätzungen bereitgehalten und auf Anfrage zur Verfügung gestellt werden, wobei bei vertraulichen Daten selbstverständlich die Vertraulichkeit gewährleistet sein muss.

Das Projekt des Journal of Money, Credit, and Banking hat gezeigt, dass empirische Ergebnisse häufiger überprüft werden sollten. Im Begutachtungsprozess der Zeitschriften geschieht dies in aller Regel nicht und kann auch nicht verlangt werden. Soweit die Daten nicht vertraulich sind, könnten solche Nachschätzungen aber z.B. interessante Aufgaben im Rahmen der Ökonometrieausbildung sein. Damit Anreize dazu bestehen, sollten die Zeitschriften offener gegenüber der Veröffentlichung von Kommentaren sein, wenn diese abweichende empirische Ergebnisse enthalten. Dies würde zudem bei den Autoren den Anreiz erhöhen, bei der Schätzung und der Dokumentation der Ergebnisse sorgfältig vorzugehen, um zu vermeiden, dass Fehler entstehen, die andere dann aufdecken. Erforderlich ist auch eine faire Begutachtung dieser Kommentare durch unabhängige Gutachter. Den bzw. einen Autor der ursprünglichen Arbeit als Gutachter einzusetzen, wie es vom Herausgeber des Journal of Political Economy im Fall Oberholzer-Gee/Stumpf vs. Liebowitz geschah und was leider kein Einzelfall ist, ist mit einer fairen Beurteilung kaum vereinbar. Dazu kommt, dass ein solcher Kommentar in einer anderen Zeitschrift kaum je unterzubringen ist und dort im Zweifelsfall auch gar nicht beachtet würde. Dies kann freilich heute durch das Internet zum Teil ausgeglichen werden. ${ }^{45}$

45. Siehe hierzu z.B. die Kommentare und Diskussionen zu in anderen Zeitschriften veröffentlichten Papieren in der Internet Zeitschrift Econ Journal Watch (http://econjwatch.org/ (07/09/12). 
Das Problem ist somit nicht, dass es grundsätzlich problematisch wäre, Gutachten gegen Bezahlung zu erstellen oder externe Drittmittel für Forschungsvorhaben zu erhalten, auch wenn es gelegentlich Sinn machen kann, von bestimmten potentiellen Auftraggebern keine Mittel mehr anzunehmen. So hat das Deutsche Krebsforschungszentrum einen Ethischen Kodex zur Ablehnung von Tabakindustriegeldern für die Krebsforschung verabschiedet, ${ }^{46}$ d.h. es nimmt, genauso wie zahlreiche Universitäten, keinerlei Forschungsgelder mehr von der Tabakindustrie an. ${ }^{47}$ Das Problem liegt auch nicht daran, dass von externen Geldgebern gesponserte Forschung grundsätzlich verzerrte Ergebnisse aufweisen müsste. Das Problem liegt in der mangelnden Transparenz möglicher Interessenkonflikte. Höhere Transparenz ist hier nicht (nur) ein Wert an sich, sondern sie zwingt auch die Betroffenen, bei ihrer Auftragsforschung besonders sorgfältig vorzugehen, um nicht in den Verdacht zu geraten, für die Geldgeber Gefälligkeitsgutachten zu erstellen. Je transparenter alle Verbindungen und damit auch alle potentiellen Interessenkonflikte offenliegen, desto wahrscheinlicher ist - zumindest bei seriösen Wissenschaftlern - ein Reputationsverlust mit Gefälligkeitsgutachten verbunden, und desto geringer ist die Gefahr, dass solche erstellt werden.

\section{Thesenartige Zusammenfassung und Schlussfolgerungen}

(i) Das ökonomische Verhaltensmodell gilt für Wissenschaftler und damit auch für wissenschaftliche Politikberater in gleicher Weise wie für alle anderen Menschen. Wir alle verfolgen unsere eigenen Interessen, aber selbstverständlich nur unter Nebenbedingungen und nicht unter allen Umständen. Eigeninteresse ist zwar nicht die einzige, aber dennoch eine sehr wichtige Motivation für das Handeln der Individuen.

(ii) Objektivität in den Wissenschaften ist vor allem ein Ergebnis eines sinnvoll organisierten Wissenschaftsprozesses und weniger Folge der Objektivität der einzelnen Wissenschaftler. Dies gilt auch für die Politikberatung. Gerade bei Politikberatern kann man davon ausgehen, dass sie auch eigene politische (ideologische) Überzeugungen haben, die für ihre Arbeiten nicht völlig ohne Belang sind.

(iii) Die ökonomische Theorie allein bietet in aller Regel keine eindeutigen Antworten auf Fragen nach der Lösung wirtschaftspolitischer Probleme. Dies gilt insbesondere dann, wenn Einkommens- und Substitutionseffekt gegenläufig sind. Daher sind empirische Analysen für den Beratungsprozess unverzichtbar.

(iv) Aber auch die empirischen Analysen liefern häufig keine eindeutigen Ergebnisse. In vielen Fällen können die ,gewünschten“ empirischen Ergebnisse durch eine entsprechende Ausgestaltung der empirischen Analysen erzielt werden, ohne dass man deshalb Betrug oder opportunistisches Verhalten unterstellen müsste.

(v) Die von den Auftraggebern gewünschten Ergebnisse können in vielen Fällen durch eine entsprechende Auswahl der Gutachter erhalten werden. Auftraggeber wählen Gutachter

46. Siehe http://www.dkfz.de/de/dkfz/satzung.html (07/9/12).

47. Siehe hierzu Grüning und Schönfeld (2007). 
mit ähnlichen politischen Vorstellungen, genau wie Gutachter sich vor allem jenen Auftraggebern zur Verfügung stellen, die ihnen politisch nahestehen.

(vi) Damit die Berater Anreize haben, in ihren Gutachten korrekte Aussagen zu machen, muss der Prozess der wirtschaftspolitischen Beratung offen und transparent organisiert werden. Dies impliziert auch, dass Wissenschaftler, die auch beratend tätig sind, dies bei ihren wissenschaftlichen Arbeiten offenlegen, soweit ein inhaltlicher Zusammenhang zwischen diesen und ihrer Beratungstätigkeit besteht.

(vii) Wichtig ist, dass die Gutachten sowie die Daten, auf denen sie basieren, öffentlich zugänglich sind und damit der Kritik ausgesetzt werden können. Basieren Gutachten auf vertraulichen Daten, dann ist sicherzustellen, dass Nachschätzungen möglich sind, wobei die Vertraulichkeit selbstverständlich zu wahren ist.

(viii) Ist der Beratungsprozess transparent und ist eine offene Diskussion möglich, kann wissenschaftliche Politikberatung auch und gerade dann, wenn mit Hilfe ökonometrischer Verfahren empirische Zusammenhänge untersucht werden, zu einer 'Verbesserung' der Politik beitragen. Dies gilt auch dann, wenn gegensätzliche Interessen im Spiel sind.

\section{Literaturangaben}

Adorno, Th.W. (1962), Die Logik der Sozialwissenschaften, Kölner Zeitschrift für Soziologie und Sozialpsychologie 14, 249-263; abgedruckt in: T.W. Adorno et al., Der Positivismusstreit in der deutschen Soziologie, Luchterhand, Neuwied 1969, 125-143.

Agell, J., T. Lindh und H. Ohlson (1997), Growth and the Public Sector: A Critical Review Essay, European Journal of Political Economy 13, 33-52.

Agell, J., T. Lindh und H. Ohlson (1999), Growth and the Public Sector: A Reply, European Journal of Political Economy 15, 359-366.

Agell, J., H. Ohlson und P.S. Thoursie (2006), Growth Effects of Government Expenditure and Taxation in Rich Countries: A Comment, European Economic Review 50, 211-218.

Anderson, R.G., und W.G. Dewald (1994), Replication and Scientific Standards in Applied Economics a Decade After the Journal of Money, Credit, and Banking Project, Federal Reserve Bank of St. Louis Review 76, Heft 6, 79-83.

Arrow, K.J. (1951), Social Choice and Individual Values, Wiley, New York, 2. Auflage 1963.

Buchanan, J.M., und G. Tullock, (1962), The Calculus of Consent: Logical Foundations of Constitutional Democracy, University of Michigan Press, Ann Arbor.

Dewald, W.G., J.G. Thursby und R.G. Anderson (1986), Replication in Empirical Economics: The Journal of Money, Credit, and Banking Project, American Economic Review 76, 587-603.

Dölling, D., und H. Entorf, D. Hermann und Th. Rupp (2009), Is Deterrence Effective? Results of a Meta-Analysis of Punishment, European Journal for Criminal Policy Research 15, 201-224.

Downs, A. (1957), An Economic Theory of Democracy, Harper and Row, New York; deutsche Übersetzung: Ökonomische Theorie der Demokratie, Mohr (Siebeck), Tübingen 1968.

Duso, T., D.J. Neven und L.-H. Röller (2007), The Political Economy of European Merger Control: Evidence using Stock Market Data, Journal of Law and Economics 50, 455-489. 
Ehrlich, I. (1975), The Deterrent Effect of Capital Punishment: A Question of Life and Death, American Economic Review 65, 397-417.

Fagan, J. (2006), Death and Deterrence Redux: Science, Law and Causal Reasoning on Capital Punishment, Ohio State Journal of Criminal Law 4, 255-320.

Ferguson, Ch.H. (2012), Predator Nation: Corporate Criminal, Political Corruption, and the Hijacking of America, Crown Business, New York.

Fölster, S., und M. Henrekson (1999), Growth and the Public Sector: A Critique of the Critics, European Journal of Political Economy 15, 337-358.

Fölster, S., und M. Henrekson (2001), Growth Effects of Government Expenditure and Taxation in Rich Countries, European Economic Review 45, 1501-1520.

Fölster, S., und M. Henrekson (2006), Growth Effects of Government Expenditure and Taxation in Rich Countries: A Reply, European Economic Review 50, 219-221.

Frankel, J. (2003), What an Economic Advisor Can Do When He Disagrees with the President, Challenge 46/3, 29-52.

Galler, H.P., und G. Wagner (eds.) (1998), Empirische Forschung und wirtschaftspolitische Beratung, Campus, Frankfurt/New York.

Gerritzen, B., und G. Kirchgässner (2012), Ideology or Facts: What determines the Results of Econometric Estimates of the Deterrence Effect of Death Penalty?, mimeo, Universität St. Gallen.

Göcke, K., (2009), Die Zukunft des Urheberrechts: Das Urteil im Pirate Bay-Verfahren, Zeitschrift für ausländisches öffentliches Recht und Völkerrecht 29, 865-882.

Grüning, Th. v., und N. Schönfeld (2007), Tabakindustrie und Ärzte: „Vom Teufel bezahlt ... “, Deutsches Ärzteblatt 12/104 vom 23. März, 770.

Hahn, R.W. (1989), Economic Prescriptions for Environmental Problems: How the Patient Followed the Doctor's Orders, Journal of Economic Perspectives 3, 95-114.

Heine, K., und K. Mause (2004), Policy Advice as an Investment Problem, Kyklos 57, 403-428.

Hermann, D. (2010), Die Abschreckungswirkung der Todesstrafe - ein Artefakt der Forschung?, in: D. Dölling, et al. (eds.): Verbrechen - Strafe - Resozialisierung. Berlin, De Gruyter, New York, 791-808.

Hesse, H. (1994): Als Wissenschaftler in der Politik?, in: Universität Hannover (ed.): Vorträge im Fachbereich Wirtschaftswissenschaften, Band 20, Hannover, 17-37.

Jones, Ph.R., und J.G. Cullis (1993), Public Choice and Public Policy: The Vulnerability of Economic Advice to the Interpretations of Politicians, Public Choice 75, 63-77.

Kirchgässner, G. (1991), Homo Oeconomicus, Das ökonomische Modell individuellen Verhaltens und seine Anwendung in den Wirtschafts- und Sozialwissenschaften, J.B.C. Mohr (Paul Siebeck), Tübingen; 3. Auflage 2008.

Kirchgässner, G. (1993), Vom Nutzen der Wirtschaftstheorie für die Wirtschaftspolitik, Konjunkturpolitik 39, 201-225.

Kirchgässner, G. (1996), Ideologie und Information in der Politikberatung: Einige Bemerkungen und ein Fallbeispiel, Hamburger Jahrbuch für Wirtschafts- und Gesellschaftspolitik 41, 9 - 41.

Kirchgässner, G. (2001), Zur politischen Ökonomie der Arbeitsmarktreformen, Volkswirtschaftliche Abteilung der Universität St. Gallen, Diskussionspapier Nr. 2001-03, März. 
Kirchgässner, G. (2004), Zum Zusammenhang zwischen staatlicher Aktivität und wirtschaftlicher Entwicklung, Abschnitt 4.1 des Jahresberichts 2004 der Kommission für Konjunkturfragen, 383. Mitteilung, Beilage zur Volkswirtschaft, dem Magazin für Wirtschaftspolitik, 1-2004, S. 42 - 48. (Hintergrundpapier:

http://www.seco.admin.ch/dokumentation/publikation/02640/02642/index.html?lang=de $(15 / 11 / 12)$.)

Kirchgässner, G. (2007), Wissenschaft und Politik: Chancen und Gefahren einer unvermeidlichen Allianz, in: W. Berka und H. Schmidinger (eds.), Vom Nutzen der Wissenschaften, Böhlau, Wien et al., 183-219.

Kirchgässner, G. (2009), Die Krise der Wirtschaft: Auch eine Krise der Wirtschaftswissenschaften?, Perspektiven der Wirtschaftspolitik 10, 436-468.

Kirchgässner, G. (2011), Der Ökonom als Berater: Objektivität, Ideologie und Eigeninteresse, in: M. HeLD, G. KUBON-GILKE und R. STURN (eds.), Ökonomik in der Krise, Jahrbuch normative und institutionelle Grundlagen der Ökonomik, Band 10, Metropolis, Marburg, 219-241.

Kirchgässner, G. (2011a), Econometric Estimates of Deterrence of the Death Penalty: Facts or Ideology?, Kyklos 64, 448-478.

Krelle, W. (1979), Schlusswort, in: C.C. v. Weizsäcker (ed.), Staat und Wirtschaft, Duncker und Humblot, Berlin, 851-855.

Kruse, J. (2010), Das Governance-Dilemma der demokratischen Wirtschaftspolitik, in: R. Ohr (ed.), Governance in der Wirtschaftspolitik, Duncker \& Humblot, Berlin, 41-71.

Lechner, M. (1989), Training the East German Labour Force: Microeconometric Evaluations of Continuous Vocational Training after Unification, Physica, Heidelberg.

Lechner, M., und M. Gerfin (2000), Wirkung der aktiven Arbeitsmarktpolitik der Schweiz auf die individuellen Beschäftigungschancen von Arbeitslosen, Beiträge zur Arbeitsmarkt- und Berufsforschung 33, 396-404.

Liebowitz, L. (2006), File Sharing: Creative Destruction or Just Plain Destruction?, Journal of Law and Economics 49, 1-28.

Liebowitz, L. (2007), How Reliable is the Oberholzer-Gee and Strumpf Paper on File-Sharing?, mimeo, University of Texas at Dallas, September, http://copyrightalliance.net/files/ssrn-id1014399.pdf (15/11/12).

Liebowitz, L. (2011), The Metric is the Message: How Much of the Decline in Sound Recording Sales is Due to File-Sharing?, mimeo, University of Texas at Dallas, November; http://www.techpolicyinstitute.org/files/the\%20metric\%20is\%20the\%20message.pdf (15/11/12).

McManus,W.S. (1985), Estimates of the Deterrent Effect of Capital Punishment: The Importance of the Researcher's Prior Beliefs, Journal of Political Economy 93, 417-425.

Manner, M., T. Siniketo und U. Polland (2009), The Pirate Bay Ruling: When the Fun and Games End, Entertainment Law Review 20, 197-205.

Mill, J.S. (1848), The Principles of Political Economy With Some of Their Applications to Social Philosophy, Vol. 2; zitiert nach der deutschen Übersetzung: Grundsätze der politischen Ökonomie, mit einigen Anwendungen auf die Sozialphilosophie, Band 2, Gustav Fischer, Jena, 1921.

Oberholzer-Gee, F., und K. Strumpf (2007), The Effect of File Sharing on Record Sales: An Empirical Analysis, Journal of Political Economy 115, 1-42.

Popper, K.R. (1962), Die Logik der Sozialwissenschaften, Kölner Zeitschrift für Soziologie und Sozialpsychologie 14, 233-248; abgedruckt in und zitiert nach: T.W. Adorno et al., Der Positivismusstreit in der deutschen Soziologie, Luchterhand, Neuwied 1969, 103-123.

Potrafke, N. (2012), Minority Positions in the German Council of Economic Experts: A Political Economic Analysis, mimeo, Universität München, Oktober. 
Schaltegger, Ch.A. (2004), Ist die Höhe der Staatsquote schuld an der Schweizer Wachstumsschwäche?, Eidgenössische Steuerverwaltung ESTV, Abteilung Steuerstatistik und Dokumentation, Bern.

Schaltegger, Ch.A., und B. Torgler (2006), Growth Effects of Public Expenditure on the State and Local Level: Evidence from a Sample of Rich Governments, Applied Economics 38, 1181-1192.

Scheide, J. (2005), Makroökonomische Theorie und wirtschaftspolitische Beratung, Die Weltwirtschaft, Heft 4, 432-448.

Schips, B. (1995): Ökonomische Argumente für wirksamen Wettbewerb auch im Versicherungszweig ,Gebäudefeuer- und Gebäudeelementarschäden`, St. Gallen, Januar.

Shepherd, J.M. (2004), Capital Punishment and the Deterrence of Crime, Written Testimony for the House Judiciary Committee, Subcommittee on Crime Terrorism, and Homeland Security, Hearing on H.R.2934, the "Terrorist Penalties Enhancement Act of 2003," April 21.

Siebert, H. (1998), Disziplinierung der nationalen Wirtschaftspolitik durch die internationale Kapitalmobilität, in: D. DuwENDAG (ed.), Finanzmärkte im Spannungsfeld von Globalisierung, Regulierung und Geldpolitik, Duncker und Humblot, Berlin, 41-67.

Sievert, O. (1979), Die Steuerbarkeit der Konjunktur durch den Staat, in: C.C. v. Weizsäcker (ed.), Staat und Wirtschaft, Duncker und Humblot, 809-846.

Smith, A. (1776), An Inquiry into the Nature and Causes of the Wealth of Nations, London; deutsche Übersetzung: Der Wohlstand der Nationen, Eine Untersuchung seiner Natur und seiner Ursachen, C.H. Beck, München 1974.

Sonnenschein, H. (1972), Market Excess Demand Functions, Econometrica 40, 549-563.

Sonnenschein, H. (1973), Do Walras Identity and Continuity Characterize the Class of Community Excess Demand Functions?, Journal of Economic Theory 6, 345-354.

Tinbergen, J. (1956), Economic Policy: Principles and Design, North-Holland, Amsterdam; deutsche Übersetzung: Wirtschaftspolitik, Rombach, Freiburg 1968.

Ungern-Sternberg, Th. v. (1994): Die kantonalen Gebäudeversicherungen: Eine ökonomische Analyse. Cahiers de recherches économiques No 9405, Département d'économétrie et économie politique, Université de Lausanne, Juni.

VanDoren, P. (1989), Should Congress Listen to Economists?, Journal of Politics 51, 319-336.

Yang, B., und D. Lester (2008), The Deterrent Effect of Executions: A Meta-Analysis Thirty Years after Ehrlich, Journal of Criminal Justice 36, 453-460.

Weber, M. (1919): Wissenschaft als Beruf, Duncker und Humblot, München/Leipzig. 